\title{
ALS-associated fused in sarcoma (FUS) mutations disrupt Transportin-mediated nuclear import
}

\author{
Dorothee Dormann ${ }^{1,2}$, Ramona Rodde ${ }^{1,2}$, \\ Dieter Edbauer', Eva Bentmann ${ }^{1,2}$, \\ Ingeborg Fischer ${ }^{3}$, Alexander Hruscha ${ }^{1}$, \\ Manuel E Than ${ }^{4}$, Ian RA Mackenzie ${ }^{5}$, \\ Anja Capell ${ }^{1,2}$, Bettina Schmid', \\ Manuela Neumann ${ }^{3}$ and \\ Christian Haass ${ }^{1,2, *}$
}

${ }^{1}$ DZNE-German Center for Neurodegenerative Diseases, Munich, Germany, ${ }^{2}$ Adolf-Butenandt-Institute, Biochemistry, LudwigMaximilians-University, Munich, Germany, ${ }^{3}$ Institute of Neuropathology, Zurich, Switzerland, ${ }^{4}$ Leibniz Institute for Age Research, Fritz-Lipmann Institute (FLI), Protein Crystallography Group, Jena, Germany and ${ }^{5}$ Department of Pathology, Vancouver General Hospital, Vancouver, British Columbia, Canada

Mutations in fused in sarcoma (FUS) are a cause of familial amyotrophic lateral sclerosis (fALS). Patients carrying point mutations in the C-terminus of FUS show neuronal cytoplasmic FUS-positive inclusions, whereas in healthy controls, FUS is predominantly nuclear. Cytoplasmic FUS inclusions have also been identified in a subset of frontotemporal lobar degeneration (FTLD-FUS). We show that a non-classical PY nuclear localization signal (NLS) in the C-terminus of FUS is necessary for nuclear import. The majority of fALS-associated mutations occur within the NLS and impair nuclear import to a degree that correlates with the age of disease onset. This presents the first case of disease-causing mutations within a PY-NLS. Nuclear import of FUS is dependent on Transportin, and interference with this transport pathway leads to cytoplasmic redistribution and recruitment of FUS into stress granules. Moreover, proteins known to be stress granule markers co-deposit with inclusions in fALS and FTLD-FUS patients, implicating stress granule formation in the pathogenesis of these diseases. We propose that two pathological hits, namely nuclear import defects and cellular stress, are involved in the pathogenesis of FUS-opathies.

The EMBO Journal (2010) 29, 2841-2857. doi:10.1038/

emboj.2010.143; Published online 6 July 2010

Subject Categories: neuroscience; molecular biology

of disease

Keywords: amyotrophic lateral sclerosis (ALS); frontotemporal lobar degeneration (FTLD); fused in sarcoma (FUS); stress granules; Transportin

\footnotetext{
${ }^{*}$ Corresponding author. DZNE-German Center for Neurodegenerative Diseases and Adolf-Butenandt-Institute, Biochemistry, Ludwig-

Maximilians-University, Schillerstrasse 44, Munich 80336, Germany. Tel.: +4 98921807 5471; Fax: + 498921807 5415;

E-mail: chaass@med.uni-muenchen.de
}

Received: 26 March 2010; accepted: 7 June 2010; published online: 6 July 2010

\section{Introduction}

ALS, also known as Lou Gehrig's disease, is an incurable, severely disabling condition that is characterized by the degeneration of both upper and lower motor neurons. Loss of motor neurons leads to progressive muscle weakening, atrophy and spasticity. The majority of patients succumb to the disease within 1-5 years after disease onset, typically because of respiratory failure (Boillee et al, 2006). Although the majority of ALS cases are sporadic (sALS), about $10 \%$ are inherited in a dominant manner (fALS) (Boillee et al, 2006; Valdmanis and Rouleau, 2008). Of these, about 5-10\% are caused by mutations in the TAR DNA-binding protein 43 (TDP-43) gene on chromosome 1 (Gitcho et al, 2008; Kabashi et al, 2008; Mackenzie and Rademakers, 2008; Sreedharan et al, 2008) or the FUS gene on chromosome 16 (Kwiatkowski et al, 2009; Vance et al, 2009). Although these genes account for only a small number of fALS cases, their gene products seem to have a crucial function in the pathogenesis of the majority of ALS cases, including sALS, as well as the related disorder frontotemporal lobar degeneration with ubiquitin-positive inclusions (FTLD-U). Both neurodegenerative diseases are characterized by the presence of neuronal and/or glial TDP-43 or FUS inclusions. TDP-43 inclusions are found in most ALS cases, with the exception of fALS caused by mutations in the $\mathrm{Cu} / \mathrm{Zn}$ superoxide dismutase (SOD1) gene (Mackenzie et al, 2007). Moreover, TDP-43-positive inclusions are also found in $>90 \%$ of FTLDU patients (Arai et al, 2006; Neumann et al, 2006), now renamed as FTLD-TDP (Mackenzie et al, 2010). FUS inclusions are present in the remaining $10 \%$ of atypical TDP-43negative FTLD-U cases (aFTLD-U) (Neumann et al, 2009a) and in other rare cases of FTLD, such as basophilic inclusion body disease (BIBD) (Munoz et al, 2009) and neuronal intermediate filament inclusion disease (NIFID) (Neumann et al, 2009b), now subsumed as FTLD-FUS (Mackenzie et al, 2010), as well as in fALS patients carrying FUS mutations (Kwiatkowski et al, 2009; Vance et al, 2009). These diseases are now commonly termed FUS-opathies (Munoz et al, 2009). The discovery of TDP-43 and FUS inclusions in both ALS and FTLD has led to the concept that ALS and FTLD are related diseases and that the same proteins are involved in their pathogenesis (Neumann et al, 2009a). This is further supported by the fact that up to $50 \%$ of ALS patients show cognitive impairment and a significant portion of FTLD patients develop motor neuron disease (Talbot and Ansorge, 2006).

Both FUS and TDP-43 are DNA- and RNA-binding proteins that shuttle continuously between the nucleus and cytoplasm (Zinszner et al, 1997b; Ayala et al, 2008) and are involved in multiple steps of gene expression, such as transcriptional regulation, pre-mRNA splicing and microRNA processing (Buratti and Baralle, 2008; Lagier-Tourenne and Cleveland, 2009). In addition, FUS has been implicated in mRNA export and mRNA transport to neuronal dendrites 
(Fujii and Takumi, 2005; Fujii et al, 2005). Although FUS and TDP-43 normally reside and function predominantly in the nucleus, pathological FUS and TDP-43 inclusions are mostly observed in the cytosol, and inclusion-bearing cells often show a reduction of nuclear staining (Arai et al, 2006; Neumann et al, 2006, 2009a; Kwiatkowski et al, 2009; Vance et al, 2009). It is completely unclear, how cytosolic FUS and TDP-43 inclusions arise and apart from p62 and ubiquitin, no other cellular markers or co-aggregating proteins have been detected within these inclusions (Neumann et al, 2006, 2007, 2009a; Kwiatkowski et al, 2009; Vance et al, 2009). As the inclusions occur predominantly in the cytosol, defects in nucleocytoplasmic transport or enhanced aggregation in the cytosol may lead to cytoplasmic mislocalization of TDP-43 and FUS. This may interfere with their physiological nuclear function or cause a toxic gain-of-function because of excessive accumulation in the cytoplasm.

For TDP-43, a classical NLS in the N-terminal domain has been identified and experimentally confirmed (Winton et al, 2008). However, none of the over 30 mutations identified in TDP-43 so far affect the NLS, and it is still unclear whether any of the mutations functionally affect nucleocytoplasmic transport. Moreover, expression of TDP-43 in yeast and neuroblastoma cells suggested that the fALS-associated mutations might increase the aggregation propensities of TDP-43 rather than impairing its nuclear transport (Johnson et al, 2009; Nonaka et al, 2009). For FUS, it has been described that some of the fALS-associated mutations in the C-terminal region lead to an accumulation of the protein in the cytosol (Kwiatkowski et al, 2009; Vance et al, 2009). However, the underlying cellular mechanism is unknown, and it is not clear whether disturbed nuclear transport or aberrant cytoplasmic aggregation of mutant proteins leads to the cytosolic redistribution of mutant FUS. A non-classical $\mathrm{R} / \mathrm{H} / \mathrm{KX}_{2-5} \mathrm{PY}-$ NLS has been predicted in the FUS C-terminal region (Lee et al, 2006). However, experimental evidence is missing that this sequence is required for nuclear import of FUS and the function of the predicted NLS is controversial. For example, a homologous motif in the related Ewing sarcoma protein (EWS), which belongs to the same transcription factor family (Law et al, 2006; Zakaryan and Gehring, 2006), was shown to be necessary, but not sufficient for nuclear import of EWS (Zakaryan and Gehring, 2006). Furthermore, an N-terminal fragment, but not a C-terminal fragment of FUS has earlier been shown to localize to the nucleus (Zinszner et al, 1997b). Nevertheless, 12 out of 22 FUS mutations identified in fALS patients are concentrated within the predicted NLS (Figure 1A) (Belzil et al, 2009; Chio et al, 2009; Kwiatkowski et al, 2009; Ticozzi et al, 2009; Vance et al, 2009; Corrado et al, 2010) and the motif is highly conserved during evolution (Figure 1B). We, therefore, assessed whether the predicted NLS was functionally relevant and whether it would be impaired by fALS-associated FUS mutations.

\section{Results}

\section{C-terminal region of FUS is necessary and sufficient for nuclear import}

To test whether the C-terminal domain of FUS is required for nuclear import, we generated a deletion mutant lacking the C-terminal 13 amino acids ( $\Delta 514-526)$ (Figure $1 \mathrm{~A})$ and analysed its localization in HeLa cells. In contrast to wildtype (WT) FUS, which was located almost exclusively in the nucleus, the deletion mutant showed a predominantly $(72 \pm 4 \%$ ) cytoplasmic localization (Figure 1C, see D for quantification). This shows that the C-terminal domain (amino acids 514-526) is necessary for efficient nuclear import of FUS. We next investigated whether the three arginine residues most frequently mutated in FUS-positive fALS patients (R521, R522, R524; see Figure 1A) are critical for nuclear import. When we changed these three arginines to alanine (R521A/R522A/R524A), the localization of the triple point mutant was indistinguishable from that of the deletion mutant ( $73 \pm 3 \%$ cytosolic; Figure $1 \mathrm{C}$ and D), showing that at least one of these arginines is essential for nuclear localization of FUS (for individual arginine mutations, see Figure 2). In addition, the two arginine residues further upstream (R514 and R518) are also required for nuclear import, as an R514A/ R518A mutant showed a predominantly $(65 \pm 5 \%)$ cytosolic localization (Figure 1C and D). These findings suggest that fALS-associated mutations may affect a functionally active NLS located in the C-terminus of FUS.

To test whether the C-terminal tail of FUS is not only necessary but also sufficient for active nuclear import, we transplanted this domain onto the C-terminus of the cytosolic reporter protein GST-GFP. Owing to its size $(55 \mathrm{kDa})$, the GSTGFP fusion protein is largely excluded from the nucleus (Figure 1E) and requires active nuclear import mediated by an NLS (Iijima et al, 2006; Terry et al, 2007). Similar to the well-characterized classical NLS of the SV40 large T antigen (SV40-NLS) (Kalderon et al, 1984), the C-terminal 13 amino acids of FUS (FUS $\left.{ }_{514-526}\right)$ mediated an almost exclusive nuclear localization of the reporter protein (Figure $1 \mathrm{E}$, see $\mathrm{F}$ for quantification). In contrast, the same amino acids arranged in random order FUS $_{514-526}$ scrambled) were not able to mediate import of GST-GFP (Figure $1 \mathrm{E}$ and F), showing that the FUS NLS activity requires a specific sequence motif rather than the random presence of several positively charged arginines. Taken together, these experiments demonstrate that the C-terminal tail of FUS is necessary and sufficient for active nuclear import and thus constitutes a bona fide NLS.

\section{fALS-associated point mutations in the C-terminal domain disrupt nuclear import of FUS}

After functionally identifying the NLS of FUS, we analysed four fALS-associated point mutations that occur within this domain at evolutionarily conserved residues (R521G, R522G, R524S, P525L; see Figure 1B) (Chio et al, 2009; Kwiatkowski et $a l, 2009)$ and asked whether they may disrupt nuclear import. Indeed, all four point mutations showed a varying degree of cytosolic accumulation, ranging from a mild mislocalization for R521G and R524S (16 $\pm 10 \%$ and $21 \pm 7 \%$ cytosolic, respectively) over an intermediate phenotype for R522G ( $45 \pm 9 \%$ cytosolic) to a severe mislocalization for P525L (65 $\pm 5 \%$ cytosolic) (Figure 2A, see B for quantification, note that mutations were ordered according to their strength/age of onset). R521H and R521C, the fALS-associated FUS mutations identified most frequently (Belzil et al, 2009; Drepper et al, 2009; Kwiatkowski et al, 2009; Ticozzi et al, 2009; Vance et al, 2009; Corrado et al, 2010; Lai et al, 2010; Suzuki et al, 2010), also caused a mild nuclear import defect, similar to R521G (Supplementary Figure S1A 
A

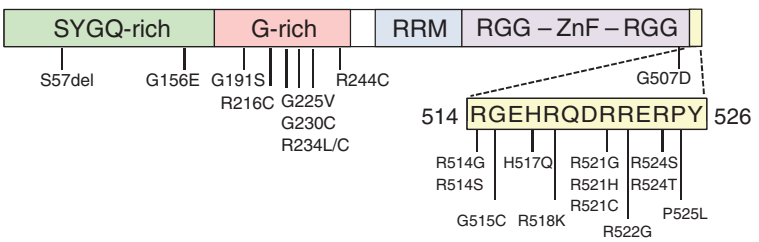

B

Human (Homo sapiens)
Chimpanzee (Pan troglodyt

Chimpanzee (Pan troglodytes)

Mouse (Mus musculus)

Rat (Rattus norvegicus)

African clawed frog (Xenopus laevis)

Zebrafish (Danio rerio)

Roundworm (Caenorhabditis elegans) - RGGPPGGD---RYRPY

Fruit fly (Drosophila melanogaster) - DGGPMRNDGGMRSRPY

C

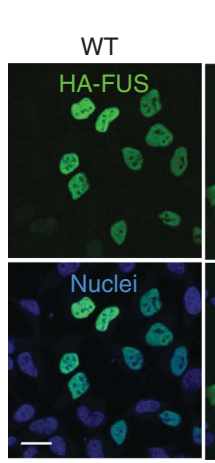

$\Delta 514-526$

R521A/R522A

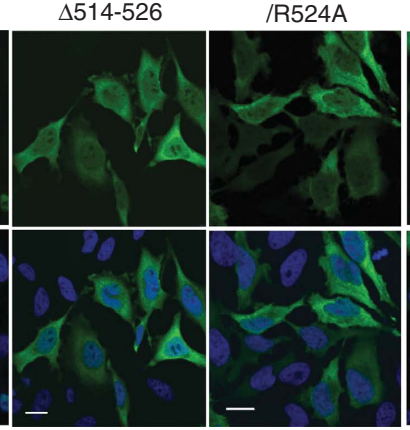

R514A/R518A

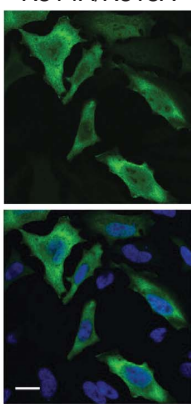

D

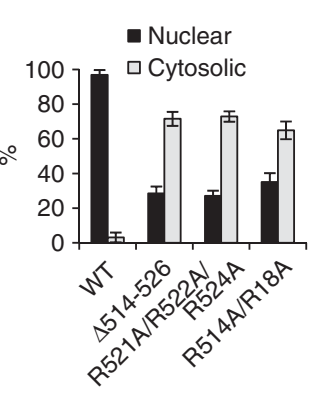

E

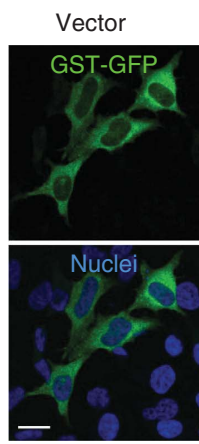

SV40-NLS
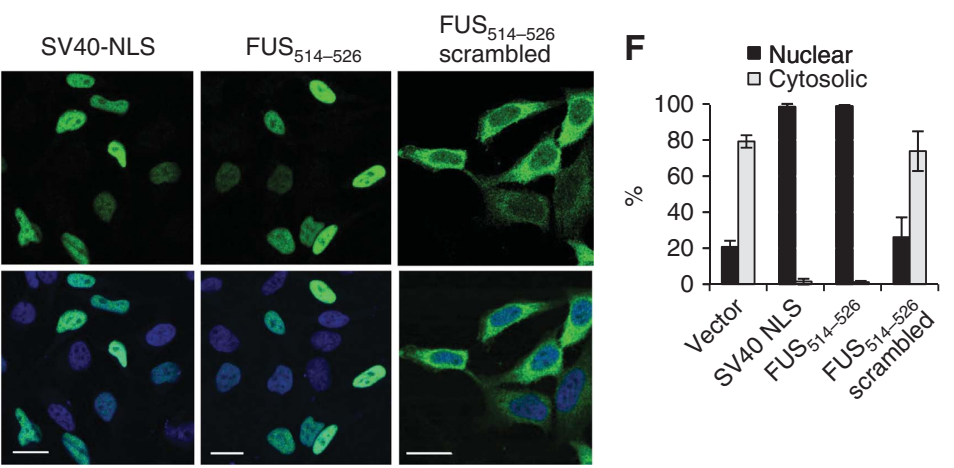

Figure 1 The C-terminal tail of FUS (FUS ${ }_{514-526}$ ) is necessary and sufficient for nuclear import. (A) Schematic diagram of the domain structure of FUS. Mutations identified in fALS patients are shown below; 12 out of the 22 known mutations are clustered in the C-terminal tail (residues 514-526). (B) Alignment of the FUS C-termini of different species shows that the sequence of this domain is well conserved during evolution (identical residues are highlighted in yellow, homologous residues in light grey). (C) N-terminally HA-tagged wild-type (WT) FUS and the indicated point mutants were transiently expressed in HeLa cells; $24 \mathrm{~h}$ post-transfection cells were stained with an HA-specific antibody (green), a nuclear counter-stain (blue) and analysed by confocal microscopy. Although the WT protein is located almost exclusively in the nucleus, deletion of the $\mathrm{C}$-terminal 13 amino acids $(\Delta 514-526)$ or replacement of arginine residues by alanine (R521A/R522A/R524A or R514A/ R518A) leads to a predominantly cytosolic localization. Scale bar, $20 \mu \mathrm{m}$. (D) Quantification of nuclear and cytosolic fluorescence intensities. Error bars indicate s.d. (E) The indicated sequences were fused to the C-terminus of the cytosolic reporter GST-GFP and reporter constructs were transiently expressed in HeLa cells; $24 \mathrm{~h}$ post-transfection cells were stained with a GFP-specific antibody (green) and a nuclear counterstain (blue) and localization of the reporter proteins was analysed by confocal microscopy. Without active nuclear import, GST-GFP is localized predominantly in the cytosol (first panel), whereas attachment of the well-characterized NLS of the SV40 large Tantigen (SV40-NLS) or the last 13 amino acids of FUS (FUS ${ }_{514-526}$ ) efficiently mediate nuclear import (second and third panel). The same amino acids arranged in random order $\left(\mathrm{FUS}_{514-526}\right.$ scrambled) are not sufficient for mediating nuclear import (last panel). Scale bar, $20 \mu \mathrm{m}$. (F) Quantification of nuclear and cytosolic fluorescence intensities. Error bars indicate s.d.

and B). The fact that none of the point mutants are completely excluded from the nucleus is consistent with the finding that in fALS patients with FUS mutations neurons with cytoplasmic FUS inclusions still show some immunolabelling of the nucleus (Kwiatkowski et al, 2009; Vance et al, 2009; Rademakers et al, 2010). The observed cytoplasmic mislocalization cannot be attributed to a higher expression level of the point mutants, as similar expression levels were observed for all mutants (Figure 2C). Thus, R522 and P525 are important residues in the C-terminal NLS of FUS, whereas R521 and R524 have a less important function for NLS activity. Another important residue is the highly conserved tyrosine at the C-terminus, which is predicted to have an important function in a PY-NLS (Lee et al, 2006), as its mutation to an alanine results in a dramatic relocalization of FUS (Supplementary Figure S1C and D). Furthermore, it is remarkable that the P525L and R522G mutations, which show the strongest degree of cytosolic mislocalization, were 
A

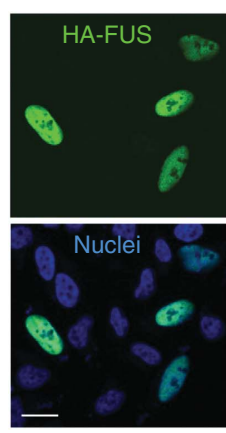

R521G

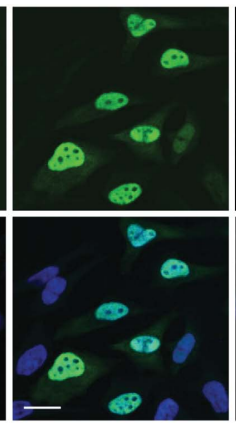

B
R524S

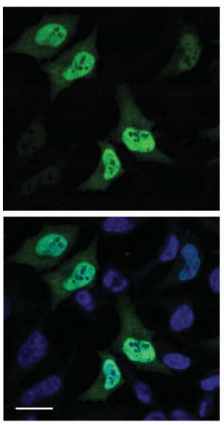

R522G

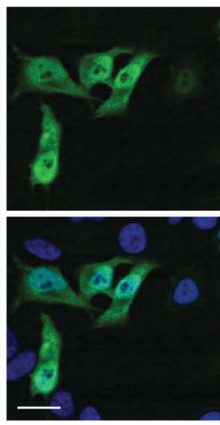

P525L

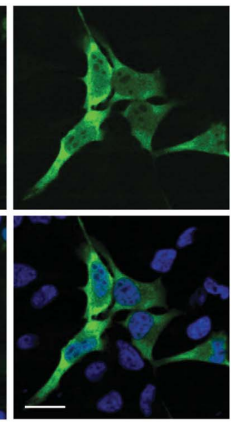

C
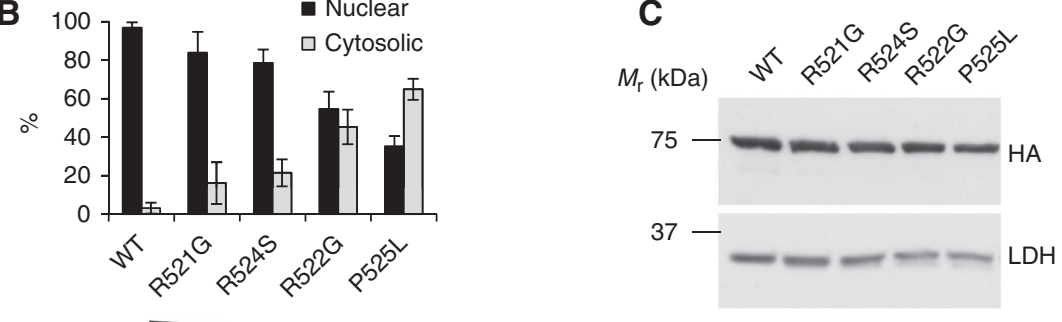

D

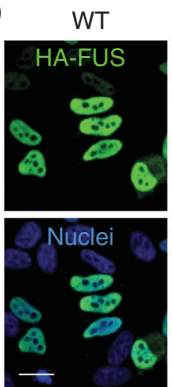

\section{G156E}
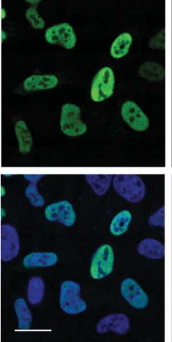
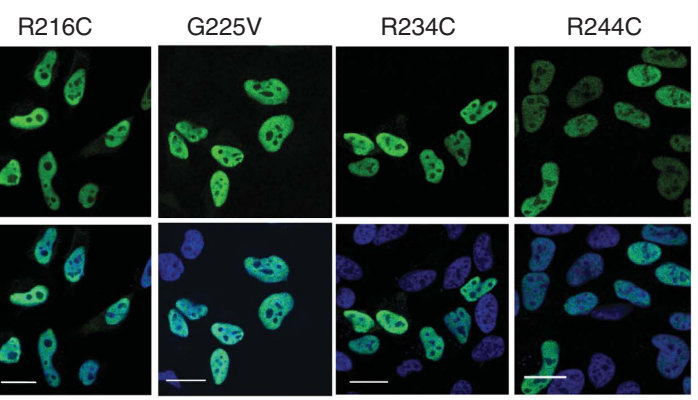

E

- Nuclear

$\square$ Cytosolic

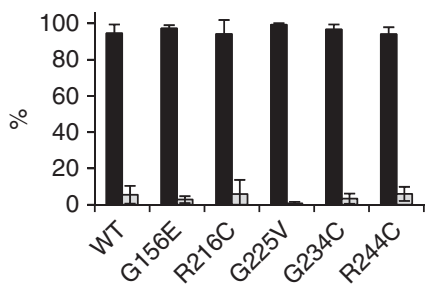

Figure 2 fALS-associated mutations in important residues of the FUS NLS disrupt nuclear import. (A) HA-tagged wild-type (WT) FUS or FUS carrying the indicated C-terminal point mutations was transiently expressed in HeLa cells. Cells were stained with an HA-specific antibody (green) and a nuclear counter-stain (blue) and were analysed by confocal microscopy. R521G and R524S show a mild, R522G and P525L a strong cytosolic mislocalization, suggesting that these mutations disrupt important residues of the FUS NLS. Scale bar, $20 \mu \mathrm{m}$. (B) Quantification of nuclear and cytosolic fluorescence intensities. Error bars indicate s.d. The degree of cytoplasmic mislocalization inversely correlates with the age of onset of the individual point mutations. (C) HA-FUS protein levels in HeLa cells transiently transfected with the indicated HA-FUS constructs were analysed by immunoblotting with an HA-specific antibody (upper panel). LDH served as a loading control (lower panel). (D) HA-tagged wild-type (WT) FUS or FUS carrying the indicated N-terminal point mutations was transiently expressed in HeLa cells. Cells were stained with an HA-specific antibody (green) and a nuclear counter-stain (blue) and were analysed by confocal microscopy. The nuclear/cytosolic distribution of the N-terminal point mutants is indistinguishable from the WT protein. Scale bar, $20 \mu \mathrm{m}$.

(E) Quantification of nuclear and cytosolic fluorescence intensities. Error bars indicate s.d.

reported to cause especially aggressive forms of fALS, with a mean disease onset at 24 and 28.5 years, respectively (Chio et al, 2009; Kwiatkowski et al, 2009). Thus, the degree of cytosolic mislocalization is inversely correlated to the age of disease onset (Figure 2B).

As deletion or mutation of the C-terminal NLS did not eliminate nuclear import completely (see Figures 1B and 2B), we investigated whether any of the point mutations located in the N-terminal SYGQ- and G-domains (Figure 1A) might affect a putative second NLS. However, all of the investigated N-terminal point mutants (G156E, R216C, G225V, R234C, R244C) showed an almost exclusive nuclear localization, indistinguishable from the WT protein (Figure 2D, see E for quantification). Furthermore, N-terminal point mutations did not further aggravate mislocalization of the P525L mutant (Supplementary Figure S2A and B), making it unlikely that they affect a second, weaker NLS, which might become important when the C-terminal NLS is impaired. Together, these findings show that C-terminal fALS-associated FUS mutations affect the protein's major NLS and thus impair its nuclear import, whereas the so far identified N-terminal FUS mutations do not impair nuclear localization. The latter suggests that these mutations cause disease through a different cellular mechanism.

\section{FUS-P525L mutation disrupts nuclear import in primary neurons and prevents import of a cytosolic reporter in vitro and in vivo}

As fALS-associated FUS mutations specifically cause degeneration of neurons in the cortex and spinal cord (Kwiatkowski et al, 2009; Vance et al, 2009), we wanted to confirm that these mutations also affect FUS import in neuronal cells. 
We, therefore, prepared primary neuronal cultures from embryonic (E19) rat hippocampus and frontal cortex and examined the subcellular localization of the WT protein and the strong P525L mutant on transfection of the corresponding cDNA constructs. As in HeLa cells, FUS-WT was located almost exclusively in the nucleus, whereas the P525L mutant was strongly redistributed to the cytosol, extending even into the neuronal processes in both cortical and hippocampal neurons (Figure 3A, see B for quantification). Thus, fALS-associated C-terminal FUS mutations disrupt nuclear
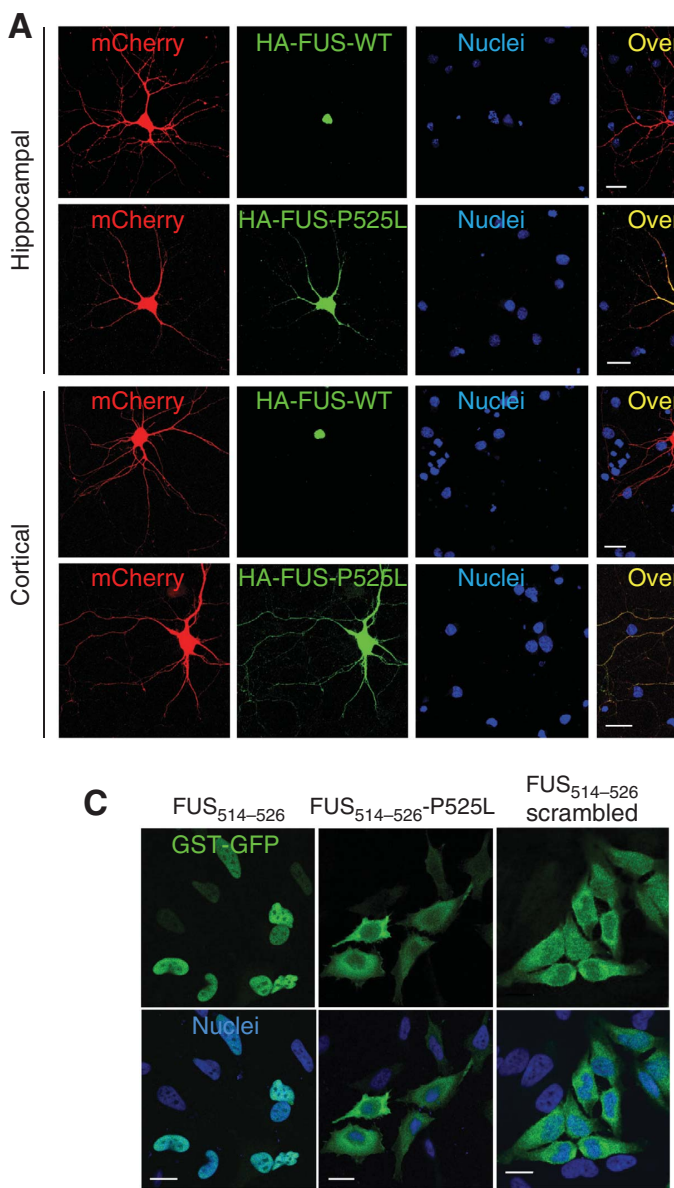
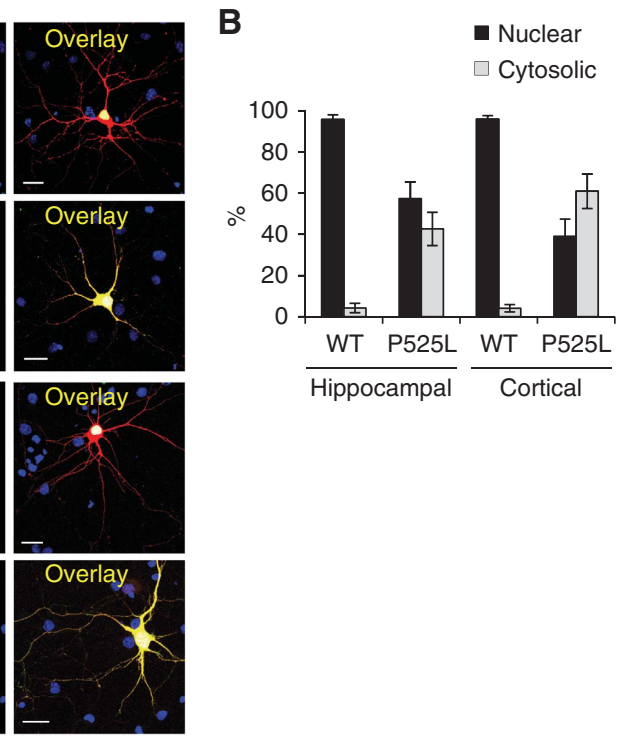

D

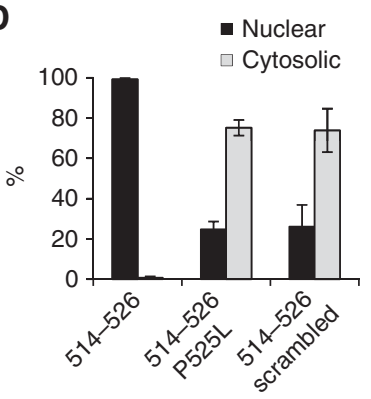

E

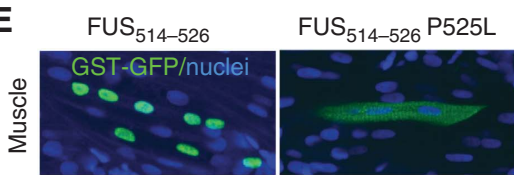

FUS $_{514-526}$

scrambled
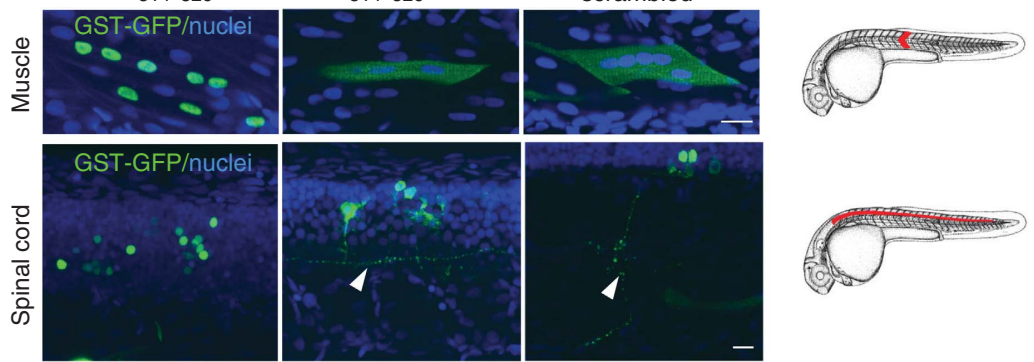

Figure 3 The FUS-P525L mutation disrupts nuclear import in primary neurons and prevents import of a cytosolic reporter in vitro and in vivo. (A) Cultured neurons from E19 rat hippocampus or frontal cortex were transfected with HA-tagged FUS-WT or P525L, mCherry (red) was coexpressed to visualize neuron morphology. Two days post-transfection, cells were stained with an HA-specific antibody (green) and a nuclear counter-stain (blue) and were analysed by confocal microscopy. FUS-WT is mostly confined to the nucleus, whereas the P525L mutant shows abundant staining in the whole cell body and in neurites. Scale bar, $20 \mu \mathrm{m}$. (B) Quantification of nuclear and cytosolic fluorescence intensities. Z-stacks were taken and all planes were projected into a single image along the $z$ axis (maximal projection). Ten fields were analysed for each sample and mean values were calculated. Error bars indicate s.d. (C) To confirm that fALS-associated point mutations disrupt nuclear import, the P525L mutation was introduced into the GST-GFP-FUS ${ }_{514-526}$ reporter and was analysed for its effect on nuclear import activity. Localization of the P525L-containing reporter is identical to that of the FUS $_{514-526}$ scrambled reporter, showing that this point mutation completely disrupts activity of the C-terminal NLS. Scale bar, $20 \mu \mathrm{m}$. (D) Quantification of nuclear and cytosolic fluorescence intensities. Error bars indicate s.d. (E) To confirm functionality of the FUS-NLS in vivo, the indicated GST-GFP reporter constructs were injected into fertilized zebrafish eggs. On day 2 post-fertilization, embryos were stained with a GFP-specific antibody (green) and a nuclear counter-stain (blue), and subcellular localization of the reporter constructs was analysed in muscle cells and spinal cord neurons by confocal microscopy. In both cell types, the FUS F14-526 $_{1}$ WT sequence mediates efficient nuclear import (left panels), whereas reporter proteins carrying the P525L mutation or scrambled NLS remain cytosolic (middle and right panels). Arrowheads indicate axonal localization of reporter proteins in spinal cord neurons. Scale bar, $10 \mu \mathrm{m}$. 
import not only in transformed cell lines, but also in primary neuronal cells, the cell type primarily affected in FUS-opathies.

To further support that cytosolic mislocalization of the fALS mutants was due to a disrupted NLS activity, the P525L mutation was introduced into the GST-GFP-FUS ${ }_{514-526}$ reporter construct and analysed for its effect on nuclear import. Consistent with the data shown in Figures 2A and $3 \mathrm{~A}$, the proline mutation efficiently blocked nuclear import of the reporter protein, as its localization was indistinguishable from that of the $\mathrm{FUS}_{514-526}$ scrambled reporter (Figure 3C and D). This further supports that fALS-associated point mutations disrupt the FUS ${ }_{514-526}$ NLS. To provide in vivo evidence for these findings, we injected the same reporter constructs into zebrafish eggs and analysed their subcellular localization in zebrafish embryos on day 2 post-fertilization. In both myocytes and spinal cord neurons, the FUS $_{514-526}$ WT sequence mediated efficient nuclear import, whereas reporter proteins carrying the P525L mutation or scrambled NLS remained cytosolic and were detectable in axonal processes of spinal cord neurons (Figure 3E). These data corroborate that fALS-associated point mutations in the FUS-NLS disrupt nuclear import in a living animal in different cell types, including spinal cord neurons.

\section{Nuclear import receptor Transportin is required for nuclear import of FUS}

Next, we searched for the cellular mechanism of nuclear transport affected by the fALS mutations described above. The NLS of FUS bears some homology to other NLSs with a PY motif, which have been shown to be recognized by the nuclear transport receptor Transportin (Trp), also known as Karyopherin $\beta 2$ (Lee et al, 2006; Imasaki et al, 2007). As FUS has been shown to interact with Trp in in vitro pull down assays (Guttinger et al, 2004; Lee et al, 2006), we speculated that Trp may mediate nuclear import of FUS by binding to its C-terminal NLS and that fALS-associated point mutations might interfere with this import pathway. To test whether Trp is responsible for nuclear import of FUS, we performed siRNA-mediated knockdown of the two Trp homologues, Trp1 and Trp2, two closely related proteins shown to have redundant function as nuclear import receptors (Guttinger et al, 2004; Rebane et al, 2004). Whereas knockdown of either Trp1 or Trp2 alone had not effect on nuclear import of FUS (data not shown), knockdown of both Trp variants significantly impaired nuclear import of endogenous FUS, as about $25 \%$ of the protein was found outside the nucleus on Trp1/2 silencing (Figure $4 \mathrm{~A}$, see B for quantification). The fact that substantial amounts of FUS are still detectable in the nucleus may be due to residual Trp1 remaining in siRNAtransfected cells (Figure 4C, middle panel) or could indicate that additional import receptors are involved in nuclear import of FUS. To test whether Trp is the predominant import receptor for the C-terminal NLS, we expressed the two FUS mutants R521G and P525L in cells that had been transfected with Trp1/2-specific siRNAs. As a control, we examined the behaviour of HA-tagged WT FUS. Similar to endogenous FUS (Figure 4A), HA-tagged FUS-WT showed a moderate redistribution to the cytoplasm on Trp knockdown (Figure 4D and $E$, see $F$ for knockdown efficiency and HA-FUS protein levels). Interestingly, the FUS-R521G mutant, which showed only a mild cytosolic mislocalization in non-siRNA transfected (Figure 2A) or control siRNA-transfected cells
(Figure 4D, upper panel), was strongly affected by Trp silencing, as $>60 \%$ of the protein redistributed to the cytosol under these conditions (Figure 4D and E). This shows that the R521G mutation indeed interferes with the Trp pathway and strongly suggests that Trp is the major import receptor for the C-terminal NLS of FUS. In line with this, the severe cytosolic mislocalization of the P525L mutant was only slightly, if at all, aggravated by Trp silencing (Figure 4D and E). This further corroborates that Trp is the major import receptor recognizing the FUS PY-NLS.

The structures of several non-classical PY-NLSs bound to Trp have been solved and converge to a consensus-binding geometry consisting of an $\mathrm{N}$-terminal hydrophobic/basic motif and the $\mathrm{C}$-terminal motif $\mathrm{R} / \mathrm{H} / \mathrm{KX}_{2-5} \mathrm{PY}$ (Lee et al, 2006; Cansizoglu et al, 2007; Imasaki et al, 2007). On the basis of these data, we modelled the FUS C-terminal sequence as subtype RXXPY bound to Trp (Figure 4G). Interestingly, our model shows two distinct binding areas within this RXXPY motif: R522 makes strong charged H-bond/ion-pair interactions with the side chain carboxylates of E509 and D543 of Trp1. The second area of tight binding comprises residues P525 and Y526. As in the experimental structures, the proline allows a particular kinked main chain geometry between these two residues, enabling a specific surface recognition, consisting of a hydrophobic pocket that engulfs the proline side chain and the phenyl ring of Y526 and an H-bond contact to Y526. In contrast, no specific interaction partner can be found for R521, resulting most likely in a somewhat flexible conformation, and only a weak attractive force between the receptor surface and R521 because of the negative electrostatic potential of the Trp surface in this region can be predicted. Similarly, R524 points away from the receptor surface and should not contribute significantly to recognition. Thus, our model is consistent with our analysis of the fALS-associated FUS mutations (Figure 2A and B), as changes in amino acids that are responsible for strong contacts between the FUS C-terminal sequence and Trp (R522 and P525) resulted in a severe cytosolic relocalization and early disease onset, whereas mutation of either R521 or R524 to glycine or serine only resulted in a mild mislocalization and later disease onset.

As siRNA-mediated silencing of Trp did not result in a complete block of the Trp pathway (see residual Trp1 levels in Figure 4C and F) and only led to a moderate cytosolic redistribution of WT-FUS, we searched for a more efficient way of blocking Trp-mediated nuclear import. To this end, we took advantage of a Trp-specific inhibitor peptide (M9M) designed to bind Trp with high affinity by joining the $\mathrm{N}$-terminal half of the hnRNP A1 NLS (called M9) with the C-terminal half of the PY-NLS of hnRNP M (Cansizoglu et al, 2007). By combining these two high-affinity-binding sites, the M9M peptide efficiently competes with natural substrates of Trp, such as hnRNP A1, HuR and hnRNP M (Cansizoglu et al, 2007). We reasoned that if Trp mediates nuclear import of FUS by binding to its C-terminal PY-NLS, the high-affinity peptide inhibitor should compete for this interaction and thus should prevent or reduce nuclear import of endogenous FUS. Indeed, when we expressed a GFP-M9M construct in cortical neurons (Figure 5A), hippocampal neurons (Supplementary Figure S3A) and HeLa cells (Figure 5B and C), the transfected cells showed a striking redistribution of endogenous FUS to the cytosol. In contrast, TDP-43, which is imported through 

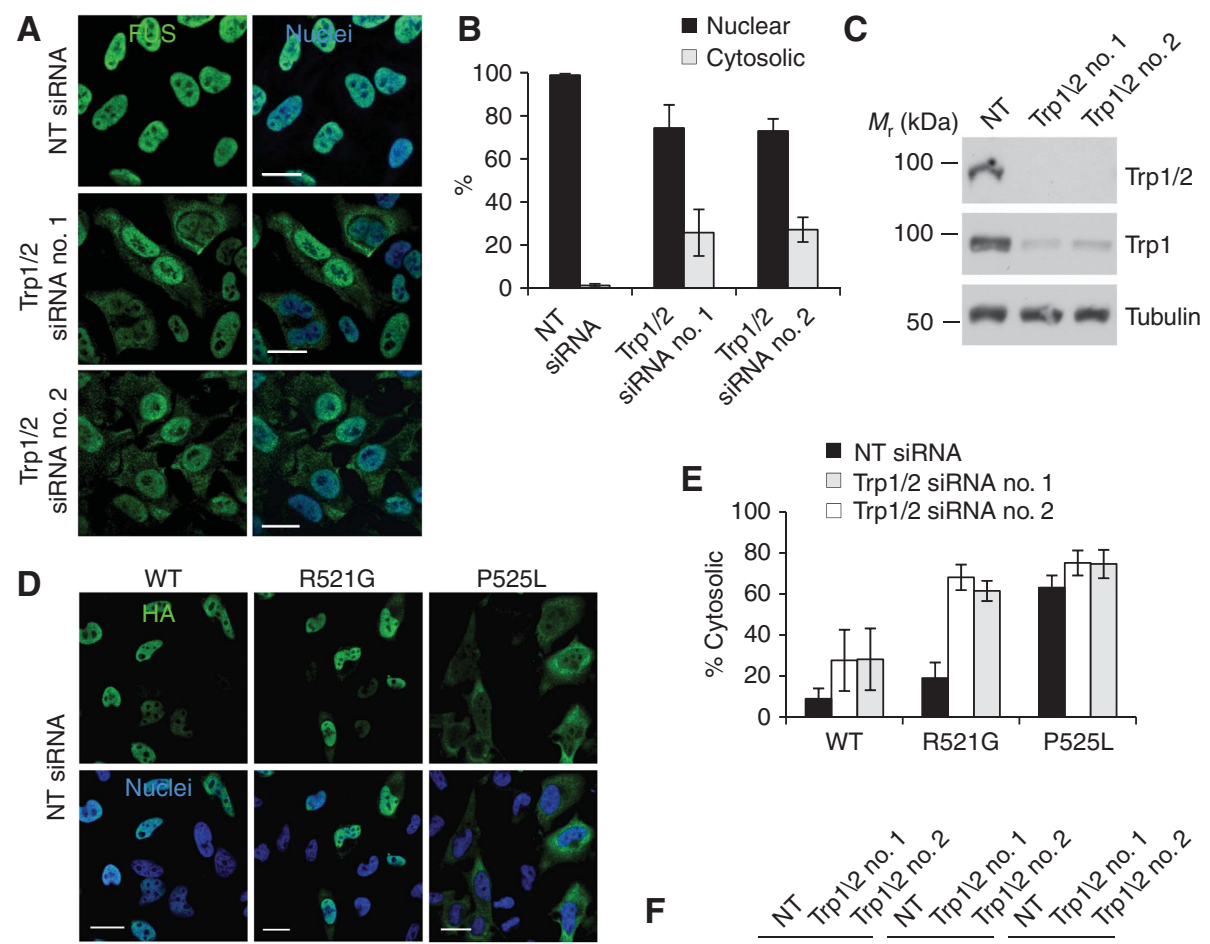

P525L
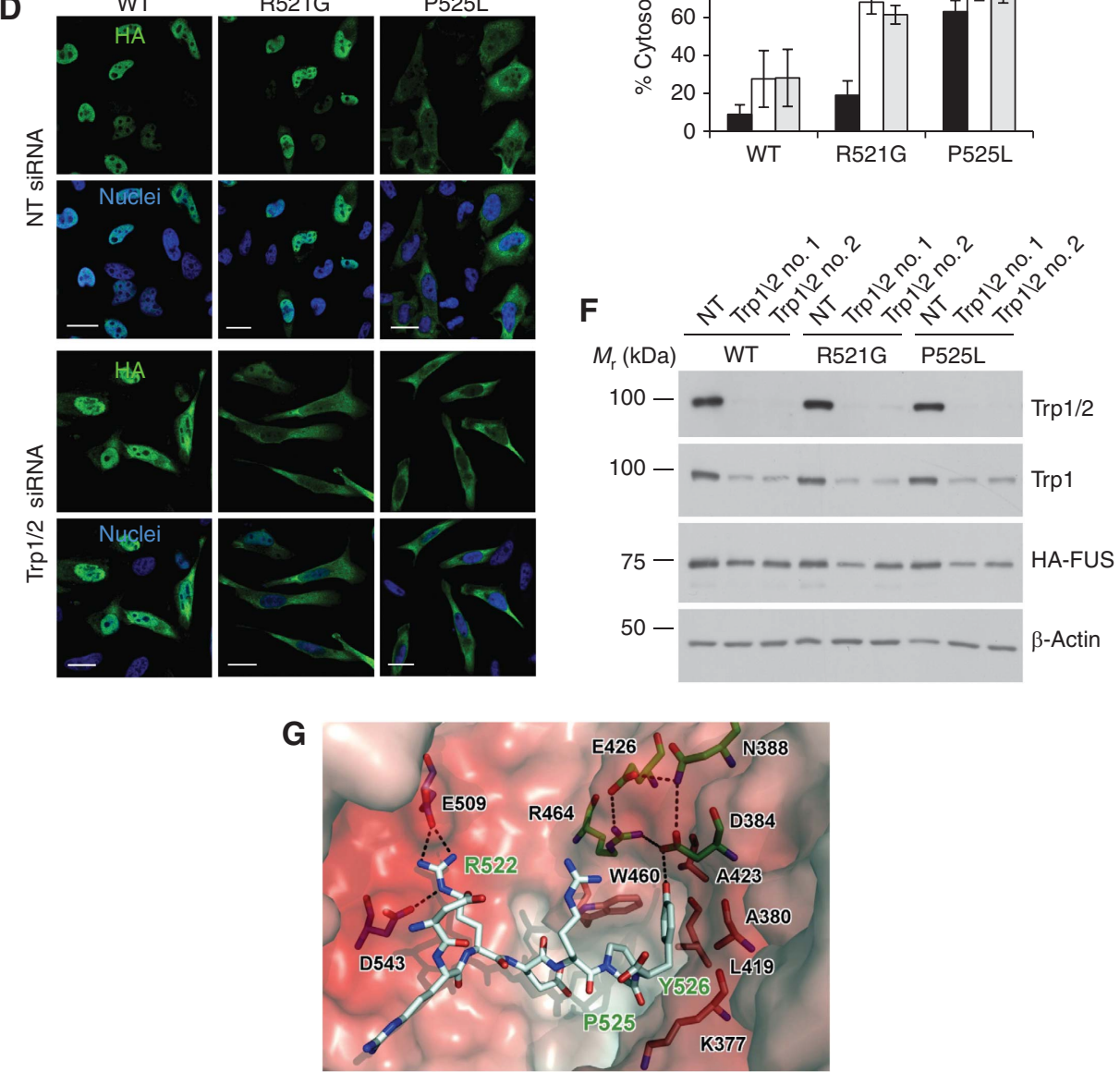

Figure 4 Transportin is required for nuclear import of FUS. (A) The two Trp homologues, Trp1 and Trp2, were silenced by siRNA-mediated knockdown, using two different siRNA pools (no. 1 and no. 2). A non-targeting (NT) siRNA was used as a negative control; $72 \mathrm{~h}$ post-transfection, cells were stained with an FUS-specific antibody (green) and a nuclear counter-stain (blue) and were analysed by confocal microscopy. Trp1/2 double knockdown leads to a partial cytoplasmic redistribution, showing that Trp is involved in nuclear import of FUS. Scale bar, $20 \mu \mathrm{m}$. (B) Quantification of nuclear and cytosolic fluorescence intensities. Error bars indicate s.d. (C) Verification of knockdown efficiency by immunoblot. Total cell lysates were examined with a pan-Trp (Trp1/2)- and a Trp1-specific antibody (upper two panels). $\alpha$-Tubulin served as a loading control (lower panel). Note that the Trp1-specific antibody is more sensitive than the pan-Trp antiserum and detects residual levels of Trp1 (middle panel). (D) HeLa cells were transfected with NT siRNA or Trp1/2-specific siRNA pool no. 1 or no. 2 and $24 \mathrm{~h}$ later with the indicated HA-tagged FUS constructs. Another $24 \mathrm{~h}$ later, cells were stained with an HA-specific antibody (green) and a nuclear counter-stain (blue) and were analysed by confocal microscopy. Trp silencing leads to a dramatic cytosolic mislocalization of the otherwise weakly mislocalized R521G mutant, but has almost no further effect on the already strongly mislocalized P525L mutant. Scale bar, $20 \mu \mathrm{m}$. (E) Quantification of nuclear and cytosolic fluorescence intensities. Error bars indicate s.d. (F) Verification of knockdown efficiency and expression of HA constructs by immunoblot. Total cell lysates were examined with antibodies specific for Trp $1 / 2$, Trp1 and HA (upper three panels). $\beta$-actin served as a loading control (lowest panel). (G) Model of the FUS PY-NLS (stick model with grey carbons, red oxygens, blue nitrogens, important amino-acid residues labelled in green) bound to the semitransparent electrostatic surface of Trp coloured according to its calculated negative ( $-25 \mathrm{e} / \mathrm{kT}$, red) and positive $(+25 \mathrm{e} / \mathrm{kT}$, blue) electrostatic surface potential. Underlying amino-acid residues of special importance for binding of the FUSNLS are depicted as stick model and labelled in black. Residues responsible for the charged H-bond/salt-bridge contact to FUS-R522 and residues forming the hydrophobic pocked for FUS-PY526 and the H-bond network connecting to FUS-Y526 OH are shown with blue, orange and green carbons, respectively. H-bonds are indicated as broken black lines. This figure was made with pymol (DeLano Scientific LLC, USA, http://www.pymol.org). 

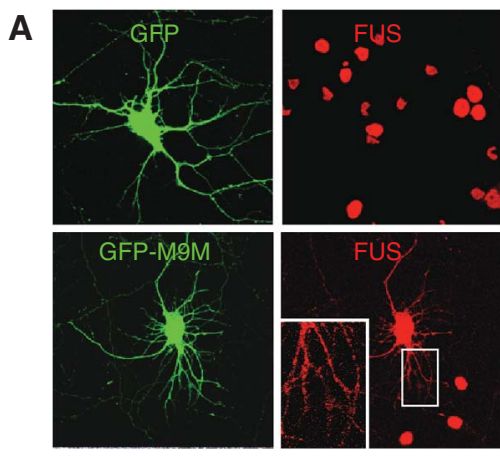

B
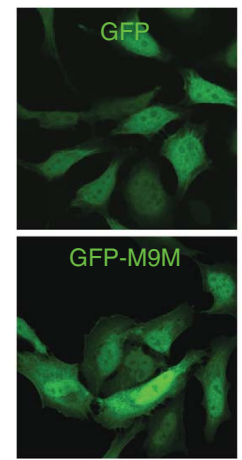

D
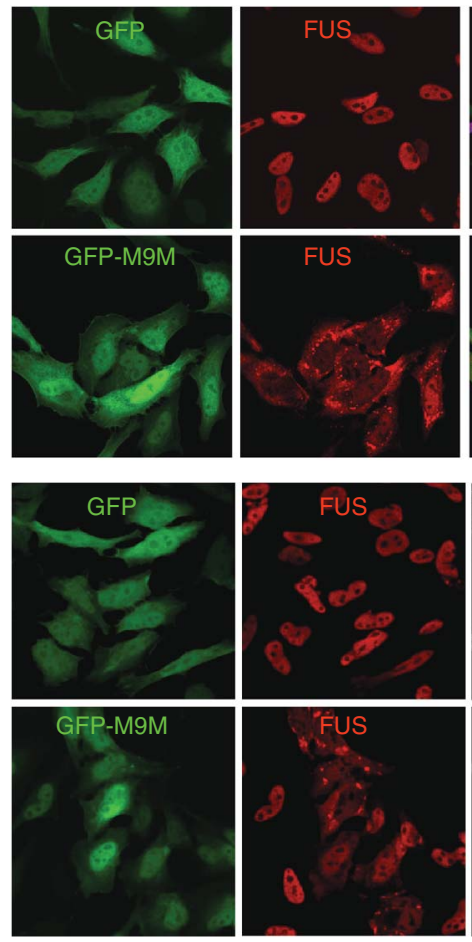
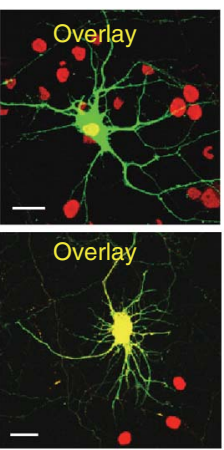

C

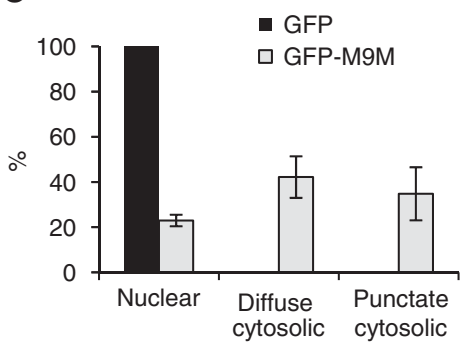

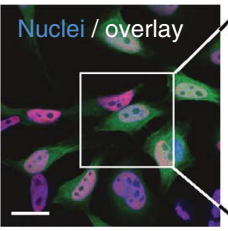
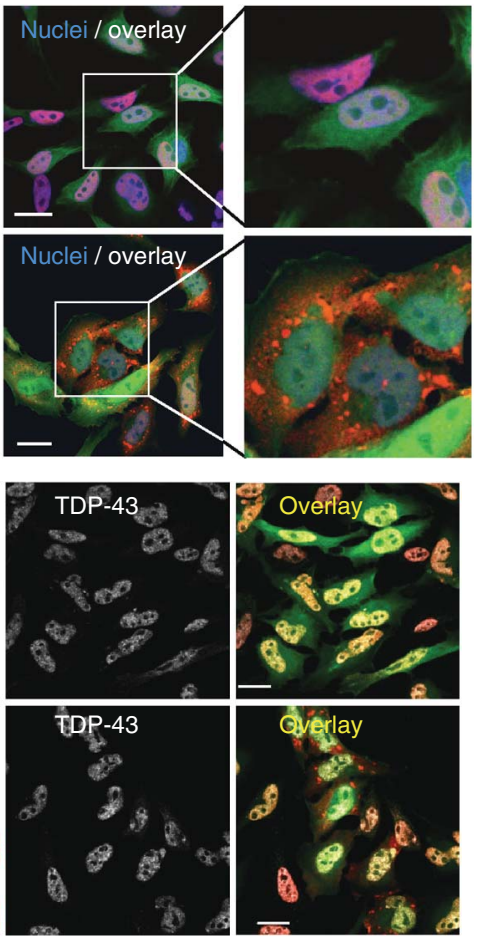

Figure 5 Expression of a Trp-specific peptide inhibitor leads to the cytosolic redistribution of FUS, but not TDP-43. (A, B) A peptide competitor (M9M) designed to bind to the PY-NLS-binding site in Trp with very high affinity was expressed in primary rat cortical neurons (A) or HeLa cells (B) as a GFP-fusion protein (green). After staining with an FUS-specific antibody (red), cells were analysed by confocal microscopy. Expression of the Trp-specific inhibitor construct causes a marked cytoplasmic redistribution and localization of endogenous FUS in cytoplasmic punctate structures. Scale bar, $20 \mu \mathrm{m}$. Insert in (A) and panels on the right of (B) show magnifications of the boxed regions. (C) Quantification of the percentage of HeLa cells with exclusively nuclear, diffuse cytosolic and punctuate cytosolic FUS staining. Error bars indicate s.d. (D) To show selectivity of the M9M peptide inhibitor, GFP or GFP-M9M (green)-transfected HeLa cells were co-stained for endogenous FUS (red) and TDP-43 (white) and were analysed by confocal microscopy. In contrast to FUS, nuclear localization of TDP-43 is not affected by expression of the M9M construct. Scale bar, $20 \mu \mathrm{m}$.

the classical NLS-dependent importin $\alpha / \beta$ (karyopherin $\alpha / \beta 1$ ) pathway (Winton et al, 2008), was not affected by expression of the M9M inhibitor and remained nuclear even in cells with cytoplasmic FUS staining (Figure 5D). As an additional control, we expressed an importin $\alpha / \beta$-specific inhibitor construct (GFP-Bimax2), designed to bind to importin $\alpha$ with high affinity (Kosugi et al, 2008). As expected, the importin $\alpha / \beta$-specific inhibitor interfered with the nuclear import of TDP-43, but not of FUS (Supplementary Figure S3B). These data show that Trp, but not the classical import receptor importin $\alpha / \beta$, is required for nuclear import of FUS. Taken together, our knockdown and competition experiments and structural modelling of the FUS PY-NLS indicate that FUS is imported into the nucleus through the Trp receptor and suggest that the pathogenic mechanism underlying the
C-terminal FUS mutations is an impairment of Trp-dependent nuclear import of FUS.

\section{Redistribution of FUS into cytoplasmic stress granules} Interestingly, we noted that after GFP-M9M expression, HeLa cells and primary neurons with cytosolic FUS redistribution often showed a punctuate localization pattern of FUS (magnifications in Figure 5A and B, see for quantification). As FUS is an RNA-binding protein (Zinszner et al, 1997b), we wondered whether the observed puncta might be stress granules, cytoplasmic RNP structures that temporarily store translationally arrested mRNAs during cellular stress (Anderson and Kedersha, 2006). In addition to stalled mRNAs, stress granules contain characteristic proteins, such as proteins of the small ribosomal subunit, translation initiation factors 
including eIF3 and eIF4G and a large variety of RNA-binding proteins, such as the PolyA-binding protein (PABP-1), the translational silencer $\mathrm{T}$ cell intracellular antigen-1 (TIA-1), TIA-1-related protein (TIAR) and the Ras-GAP-SH3-binding protein (G3BP1). These proteins specifically associate with stress granules and not with other types of cytoplasmic RNA granules, such as processing bodies (P bodies) associated with mRNA decay (Kiebler and Bassell, 2006). Therefore, they are commonly used as specific markers of stress granules (Anderson and Kedersha, 2008). Co-staining with antibodies specific to PABP-1, TIA-1, TIAR and G3BP1 showed that indeed FUS was localized to stress granules in GFP-M9Mtransfected cells (Figure 6A, rows 1-4). In contrast,
A
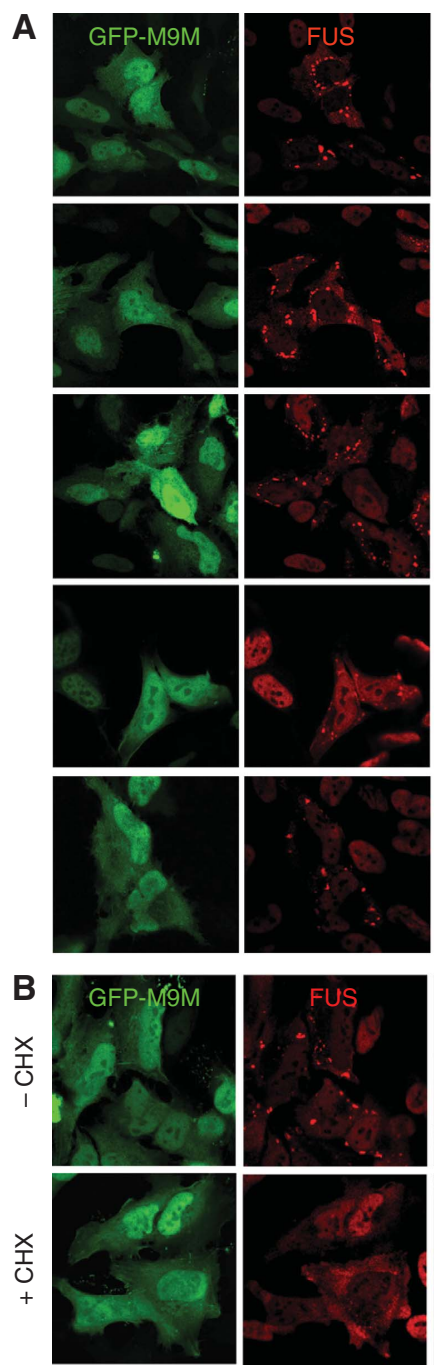

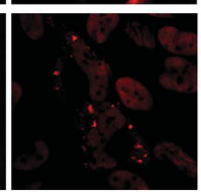

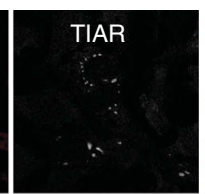
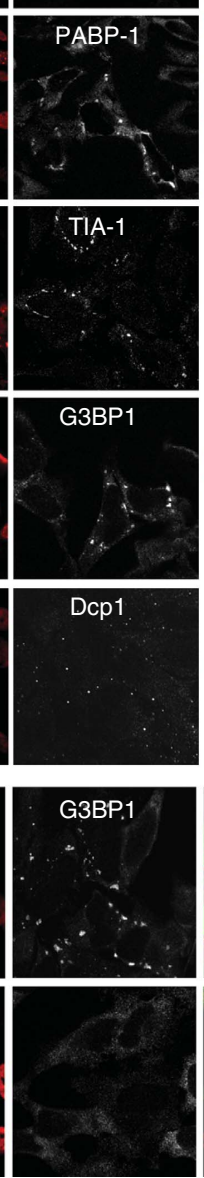
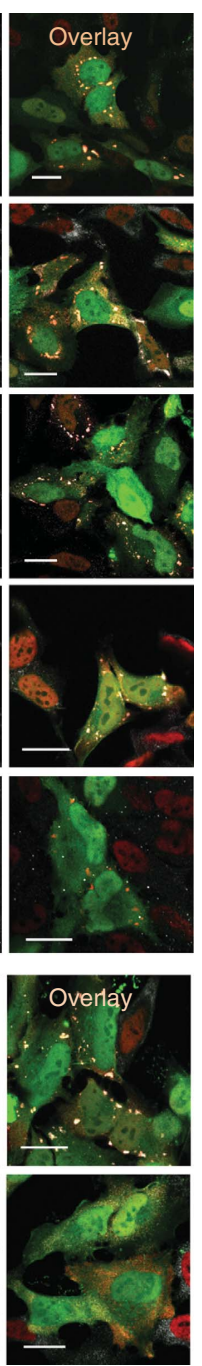

Figure 6 Redistribution of FUS into cytoplasmic stress granules. (A) GFP-M9M (green)-transfected HeLa cells were co-stained for endogenous FUS (red) and the stress granule marker proteins TIAR, PABP-1, TIA-1, G3BP1 or the P body marker Dcp1 (white). Co-staining of FUS with TIAR, PABP-1, TIA-1 and G3BP1 shows that the punctate FUS-positive structures are stress granules. Note that there is no co-localization with the P body marker Dcp1. Scale bar, $20 \mu \mathrm{m}$. (B) GFP-M9M (green)-transfected HeLa cells were stained for endogenous FUS (red) and the stress granule marker G3BP1 (white). Where indicated, the polysome-stabilizing drug cycloheximide (CHX) was added for $1 \mathrm{~h}$ before fixation to prevent stress granule formation. Cycloheximide prevents formation of G3BP1- and FUS-positive cytosolic structures, confirming their stress granule identity. Scale bar, $20 \mu \mathrm{m}$. the $\mathrm{P}$ body-specific marker protein Dcp1 did not co-stain with FUS (Figure 6A, row 5), excluding that the FUS-positive granules observed after GFP-M9M expression correspond to P bodies. Furthermore, on addition of the polysome-stabilizing drug cycloheximide, a well-known inhibitor of stress granule assembly (Kedersha and Anderson, 2007), FUS remained diffusely distributed in the cytosol and no FUS-positive G3BP1-positive granules could be observed (Figure 6B). This confirms that the FUS-positive granules observed after GFP-M9M expression are stress granules. Together, our data suggest that cytoplasmically mislocalized FUS may be recruited into stress granules under conditions of cellular stress, such as strong inhibition of Trp-dependent transport by GFP-M9M over-expression.

\section{Pathologic inclusions in fALS and FTLD-FUS patients contain marker proteins of stress granules}

As stress granules dynamically grow and coalesce on prolonged stress exposure (Kedersha et al, 2000), we wondered whether the FUS-containing stress granules might be related to the large FUS-positive inclusions present in brains of fALS and FTLD-FUS patients. We, therefore, analysed sections of post-mortem brain and spinal cord tissue from an fALS case carrying an FUS-R521C mutation and from cases with sporadic FTLD-FUS, including aFTLD-U $(n=3)$, NIFID $(n=3)$ and BIBD $(n=1)$, by immunohistochemistry and double-label immunofluorescence for the presence of the stress granule marker PABP-1 in neuronal cytoplasmic inclusions (NCIs). Strikingly, all cases with FUS pathology revealed strong labelling for PABP-1 in NCIs in affected brain regions such as spinal cord and hippocampus (Figure 7A, upper panels). This was further confirmed by double-label immunofluorescence with anti-p62 (green), a robust marker of NCIs in FUSopathies (Neumann et al, 2009a, b; Munoz et al, 2009), and anti-PABP-1 (red), which showed a clear co-localization in NCIs of all tested FUS-opathy cases (Figure 7A, lower panels). Furthermore, NCIs in all cases examined showed an enrichment for another stress granule marker protein, eIF4G (Figure 7B). Notably, cases with FTLD-TDP pathology $(n=2)$ included as neurologic controls showed no staining of NCIs with PABP-1 or eIF4G (Figure 7A and B). This suggests that co-sequestration of stress granule-associated proteins is a specific feature of FUS inclusions and that stress granule formation might be involved in inclusion body formation in FUS-opathies.

\section{C-terminal fALS mutations favour recruitment of FUS into stress granules}

It is important to note that in our cellular models FUS-positive stress granules were observed after GFP-M9M expression (Figure 6), but not on expression of mutations interfering with the NLS of FUS, despite their substantial redistribution to the cytosol (Figures 1 and 2). This suggests that cytosolic mislocalization by itself is not sufficient for the formation of FUS-containing granules, but that additional cellular stress (e.g. strong inhibition of Trp-dependent transport by GFP-M9M expression) is required for this to occur. To test this hypothesis, we expressed FUS-WT and the above-examined C-terminal FUS mutants (R521G, R522G, R524S, P525L) in HeLa cells (Figure 8A) and primary neurons (Figure 8B), subjected them to heat shock and analysed 


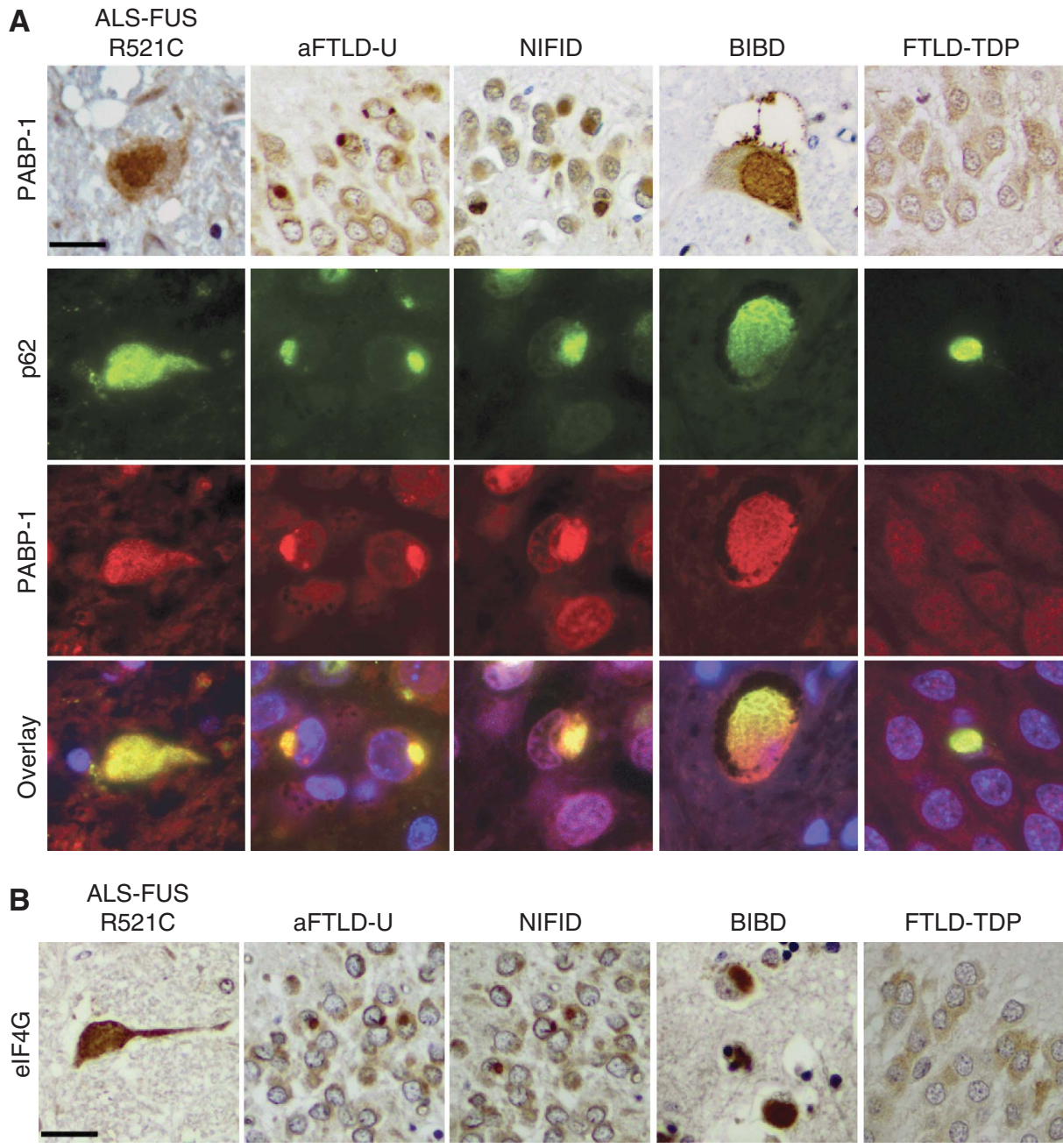

Figure 7 Neuronal cytoplasmic inclusions (NCIs) in patients with FUS pathology contain the stress granule marker proteins PABP-1 and eIF4G. (A) Upper panels: PABP-1 immunohistochemistry performed on sections of post-mortem tissue reveals strongly immunoreactive NCIs in motor neurons in the spinal cord in fALS-R521C, in dentate granule cells of the hippocampus in aFTLD-U and NIFID as well as in motor neurons in the spinal cord in BIBD. In contrast, no PABP-1-labeled inclusions were detectable in dentate granule cells of the hippocampus in FTLD-TDP. Scale bar, $25 \mu \mathrm{m}$. Lower panels: double-label immunofluorescence stainings of the same cases and brain regions show co-localization of PABP-1 (red) with p62-positive inclusions (green) in fALS-R521C, aFTLD-U, NIFID and BIBD, but no PABP-1 staining in FTLD-TDP inclusions. Note that p62 is a robust marker of FUS and TDP-43 NCIs and was used because double labelling for FUS and PABP-1 was technically not possible, as available antibodies working on paraffin-embedded tissue were both rabbit polyclonal antisera. Scale bar, $12.5 \mu \mathrm{m}$. (B) eIF4G immunohistochemistry reveals labelling of NCIs in motor neurons in the spinal cord in fALS-R521C, in dentate granule cells of the hippocampus in aFTLD$\mathrm{U}$ and NIFID and neurons in frontal cortex in BIBD. No NCIs were detectable in dentate granule cells in FTLD-TDP. Scale bar, $25 \mu \mathrm{m}$.

whether the proteins would be recruited to stress granules. In the absence of heat stress, the expressed proteins showed the above-described localization pattern and no stress granules could be detected with a PABP-1 or TIAR antibody (Figure $8 \mathrm{~A}$ and $\mathrm{B}$, left panels). In contrast, after heat shock, all FUS mutants localized to cytoplasmic stress granules (Figure $8 \mathrm{~A}$ and $\mathrm{B}$, right panels, for additional stress granule markers, see Supplementary Figure S4A), confirming our hypothesis that additional cellular stress is required for the recruitment of FUS into stress granules. The presence of cycloheximide during heat shock completely prevented the granular localization of the FUS-P525L mutant (Supplementary Figure S4B), confirming that the FUS mutant-containing granules observed after heat shock correspond to stress granules. Consistent with the GFP-M9M experiment (Figure 5D) and the pathology data shown in Figure 7, TDP-43 was not detectable in FUS-P525L-positive stress granules after heat shock (Figure 8C). Interestingly, FUS-WT also remained exclusively nuclear after heat shock and was not detectable in cytoplasmic stress granules (Figure $8 \mathrm{~A}$ and $\mathrm{B}$, right panels). This suggests that only cytoplasmically mislocalized FUS is recruited into stress granules. In line with this, the amount of mutant FUS in stress granules correlated with the degree of cytoplasmic mislocalization of the point mutants and inversely with their age of disease onset (Figure 8A). This suggests that cytosolic mislocalization strongly facilitates the formation of FUS-positive stress granules.

In conclusion, our data suggest that two pathological hits, namely cytosolic mislocalization of FUS and cellular stress, are required for the formation of FUS-positive stress granules, both in peripheral and neuronal cells (Figure 9). Furthermore, recruitment of cytoplasmic FUS into stress granules might be an important cellular mechanism leading 
A
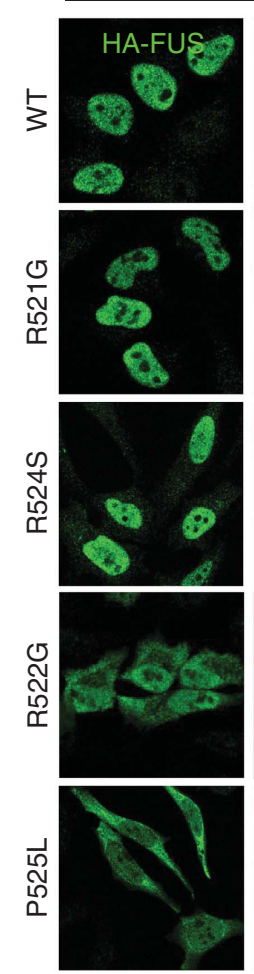

B
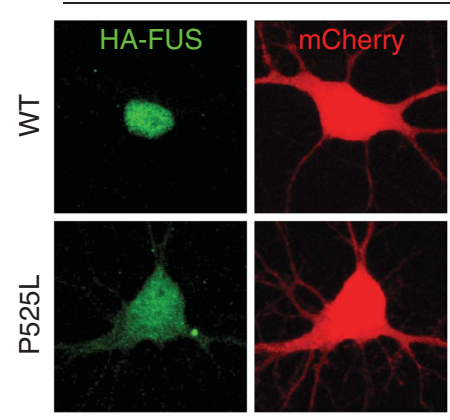

C

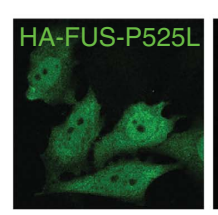

$37^{\circ} \mathrm{C}$
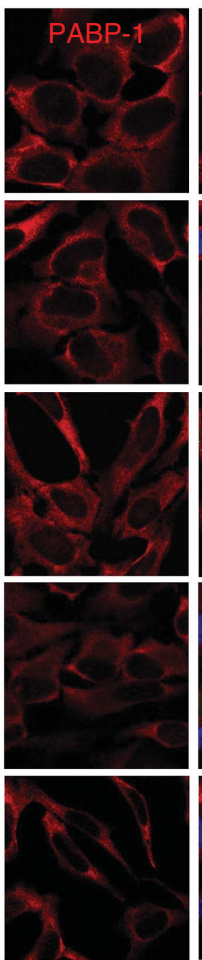

$37^{\circ} \mathrm{C}$
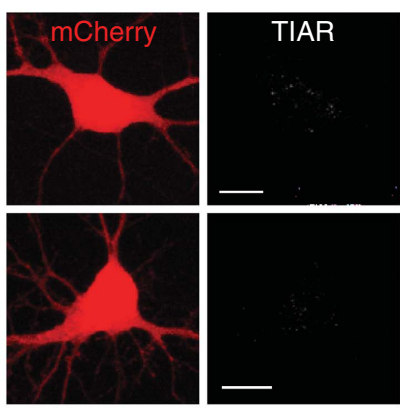

$37^{\circ} \mathrm{C}$
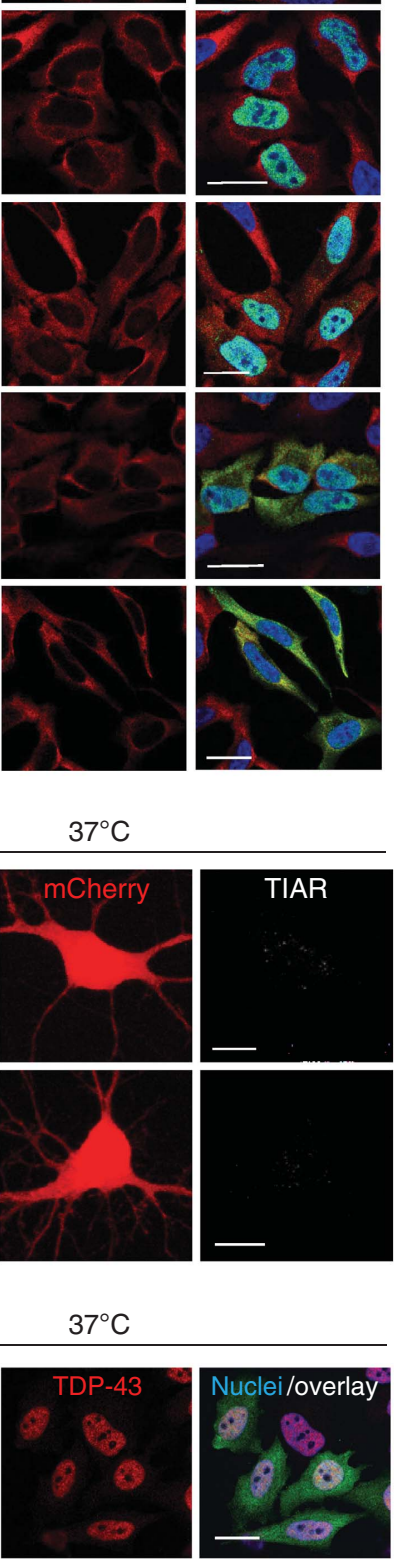
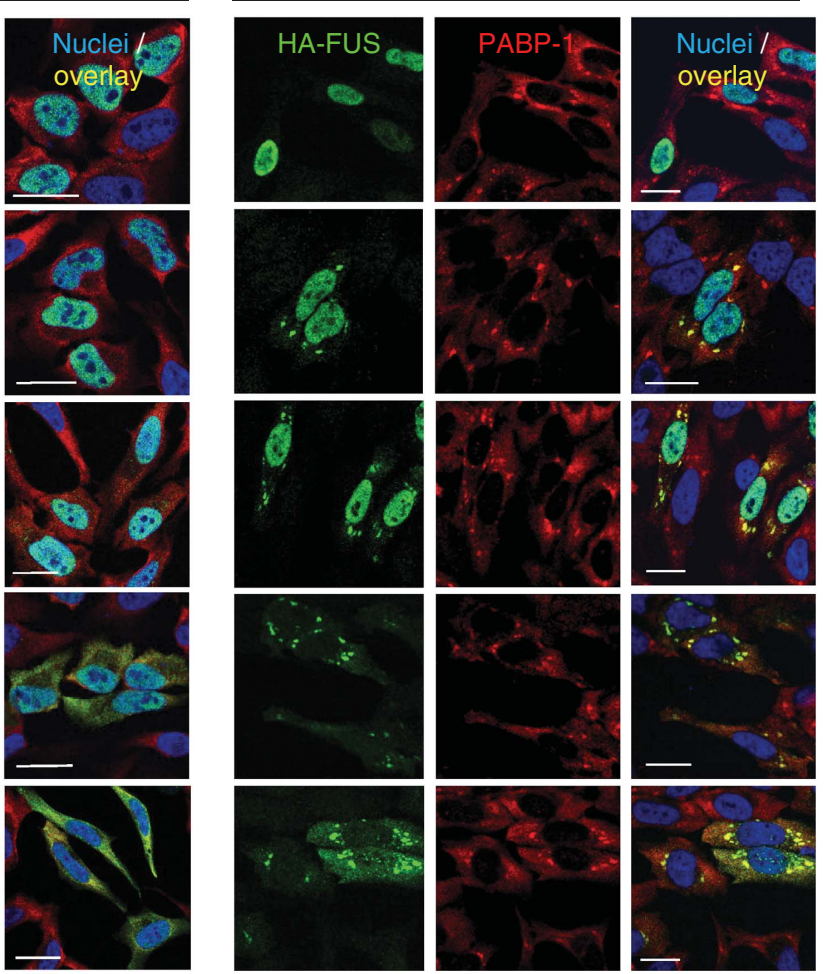

$44^{\circ} \mathrm{C}$
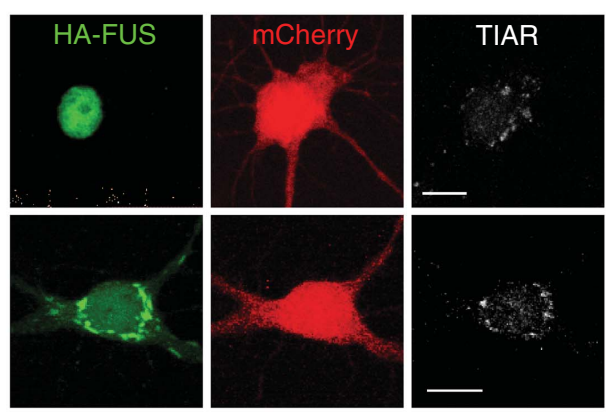

$44^{\circ} \mathrm{C}$

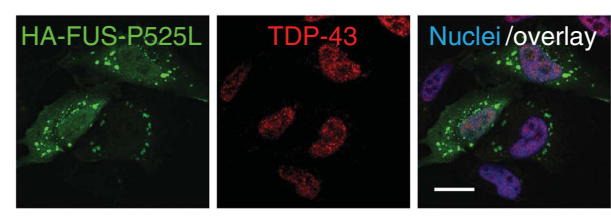

Figure 8 C-terminal fALS-associated FUS mutations favour recruitment of FUS into stress granules. (A) HeLa cells were transiently transfected with the indicated HA-tagged FUS constructs; $24 \mathrm{~h}$ post-transfection, cells were subjected to heat shock $\left(44^{\circ} \mathrm{C}\right.$ for $1 \mathrm{~h}$, right panels) or were kept at control temperature $\left(37^{\circ} \mathrm{C}\right.$, left panels). Cells were fixed, stained with an HA-specific antibody (green), a PABP-1-specific antibody (red) and a nuclear counter-stain (blue) and analysed by confocal microscopy. In contrast to WT-FUS, which remains almost exclusively nuclear on heat shock, all FUS mutants are recruited into PABP-1-positive stress granules. The amount of FUS in stress granules correlates with the cytoplasmic mislocalization and average age of disease onset of the individual point mutations, suggesting that cytoplasmic mislocalization favours recruitment of FUS to stress granules. Scale bar, $20 \mu \mathrm{m}$. (B) Primary rat hippocampal neurons were transiently transfected with HA-tagged FUSWT or the P525L mutant, mCherry (red) was co-transfected to visualized neuron morphology. Two days post-transfection, cells were subjected to heat shock $\left(44^{\circ} \mathrm{C}\right.$ for $\left.1 \mathrm{~h}\right)$ or were kept at control temperature $\left(37^{\circ} \mathrm{C}\right)$ and were stained with an HA-specific antibody (green) and a TIARspecific antibody (white). WT-FUS remains almost exclusively nuclear on heat shock, whereas the P525L mutant shows a mostly granular localization and co-localizes with TIAR-positive stress granules. Scale bar, $10 \mu \mathrm{m}$. (C) HeLa cells transiently transfected with the HA-tagged FUS-P525L mutant were subjected to heat shock $\left(44^{\circ} \mathrm{C}\right.$ for $\left.1 \mathrm{~h}\right)$ or were kept at control temperature $\left(37^{\circ} \mathrm{C}\right)$. Cells were stained with an $\mathrm{HA}$ specific antibody (green), a TDP-43-specific antibody (red) and a nuclear counter-stain (blue) and analysed by confocal microscopy. TDP-43 is not recruited into FUS-P525L-containing stress granules. Scale bar, $20 \mu \mathrm{m}$. 

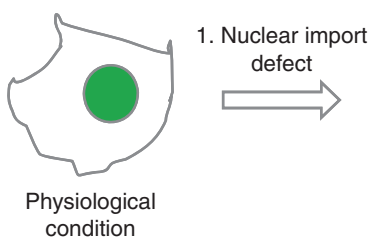

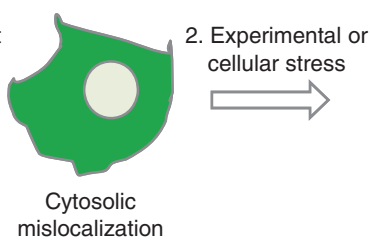

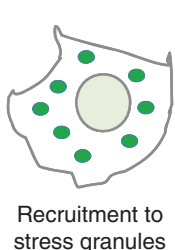

stress granules

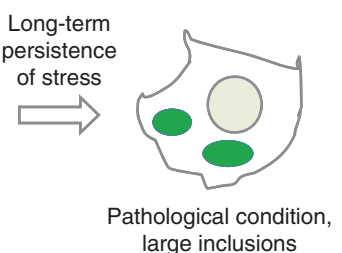

Figure 9 A two hit model of FUS pathology. Green colour represents FUS distribution. For details see Discussion.

to FUS pathology, as stress granule marker proteins also co-deposit with FUS in brains of fALS and FTLD-FUS patients (Figure 7).

\section{Discussion}

Our data show that the C-terminal domain of FUS harbours the protein's major NLS, which mediates Trp-dependent nuclear import. Furthermore, we show that several fALSassociated point mutations in the C-terminus of FUS disrupt this import mechanism, leading to cytoplasmic mislocalization of the protein. As the loss of nuclear import correlates with the age of disease onset of the individual point mutations, it seems likely that the nuclear import defect is causally linked to the disease. However, we could also show that additional cellular stress, such as heat shock, is necessary to cause a clustering of cytosolic FUS in the form of stress granules. As we consistently found the stress granule markers PABP-1 and eIF4G co-deposited with FUS inclusions in the whole spectrum of FUS-opathies, including fALS-FUS, aFTLD-U, NIFID and BIBD, we implicate cellular stress in the pathogenesis of these FUS-opathies and propose that two pathological hits, namely a nuclear import defect and cellular stress, are involved in the pathogenesis of FUS-associated diseases.

The identified NLS in the C-terminus of FUS corresponds to the class of non-classical PY-NLSs, which are recognized by the nuclear transport receptor Trp (Lee et al, 2006). PY-NLSs typically are 20-30 residue signals with a C-terminal $\mathrm{R} / \mathrm{H} / \mathrm{KX}_{2-5} \mathrm{PY}$ consensus motif preceded by a hydrophobic or basic motif, which both make important contacts with Trp (Lee et al, 2006; Imasaki et al, 2007). The C-terminal tail of FUS fulfils the criteria of this consensus motif and indeed we could show that import of FUS depends on Trp (Figures 4 and 5). Our mutational analysis suggests that R522, P525 and Y526 are the important residues of the $\mathrm{R} / \mathrm{H}-\mathrm{X}_{2-5} \mathrm{PY}$ consensus motif and that R514 and/or R518 may constitute the $\mathrm{N}$-terminal basic motif required for interaction with Trp (Figures 1 and 2; Supplementary Figure S1). This is supported by our three-dimensional model of the FUS-NLS bound to Trp (Figure 4G), explaining why mutations in residues R522, P525 and Y526 specifically affect Trp interaction and, therefore, show the most severe impairment of nuclear transport. To our knowledge, this is the first case in which mutations within a PY-NLS cause a human disease. The only other known example of disease-causing mutations in an NLS is Swyer syndrome, in which mutations in the classical NLS of SRY, the testes-determining transcription factor encoded by the human Y chromosome, lead to a reduced activation of testes-specific genes and thus male-tofemale sex reversal (Li et al, 2001; Harley et al, 2003). Considering the large number of important nuclear proteins, it seems likely that other human diseases can be attributed to nuclear import defects because of mutations within different types of NLSs.

Our data show that mutations in R522 and P525 lead to a strong cytosolic mislocalization of FUS, however, without a complete exclusion of mutant proteins from the nucleus (Figure 2A and B). This is consistent with the observations that neurons of fALS patients with FUS mutations still show some immunolabelling in the nucleus (Kwiatkowski et al, 2009; Vance et al, 2009; Rademakers et al, 2010). Although the reported number of patients is still small, it is striking that mutations in R522 and P525 cause an especially aggressive form of ALS. The five reported patients with a P525L mutation all had a very early onset of disease (mean age of onset: 24 years) and an unusually rapid disease progression, with death in $<12$ months (Chio et al, 2009; Kwiatkowski et al, 2009). R522G $(n=2)$ was the second most aggressive mutation reported by Kwiatkowski et al (2009) (mean age of onset: 28.5 years; mean duration: 25 months). Thus, the two mutations that showed the most severe nuclear import defect (Figure 2A and B) and were most readily recruited into stress granules on heat shock (Figure 7A) caused the most aggressive disease course of all reported FUS mutations. Furthermore, the R521G $(n=19)$ and R524S $(n=1)$ mutations, which showed a much weaker import defect (Figure $2 \mathrm{~A}$ and $\mathrm{B}$ ) and were recruited into heat-induced stress granules to a lesser degree (Figure 7A), on average caused disease at a much later age (average age of onset: 43 and 34 years, respectively). Interestingly, the weak R521G mutation showed incomplete penetrance, as two members of an R521G family lived well past the average age of onset without developing disease (Kwiatkowski et al, 2009). This is consistent with our hypothesis that environmental stress contributes to disease initiation. Different exposure of individuals to environmental stress might also explain the variation in the age of onset reported for other fALS-associated FUS mutations (Kwiatkowski et al, 2009). Although the number of FUS mutation carriers reported to date is still small, Alzheimer's disease (AD) research has shown that the in vitro effects of presenilin mutations can be clearly correlated with the age of disease onset (Duering et al, 2005; Page et al, 2008), and in some cases, cell culture experiments have even predicted familial AD mutations and their disease onset. Given the severe effect of the Y526A mutation on nuclear import of FUS, it seems possible that fALS patients carrying FUS-Y526 mutations may be identified in the near future and one would predict a similarly early age of onset as for the P525L mutation. Consistent with this hypothesis, a novel frameshift mutation, which leads to a premature stop codon and thus truncation of the C-terminal 60 amino acids of FUS, was recently reported with a disease onset of 20 years (DeJesus-Hernandez et al, 2010). 
In addition to mutations in the C-terminal tail of FUS, several point mutations have been identified in the N-terminal SYGQ- or G-rich domains of FUS (Belzil et al, 2009; Kwiatkowski et al, 2009; Ticozzi et al, 2009; Corrado et al, 2010) (Figure 1A). As deletion of the C-terminal NLS did not completely eliminate nuclear import (Figure 1C and D) and an N-terminal fragment comprising the SYGQ- and G-domains was described to localize to the nucleus (Zinszner et al, 1997a,b), it seemed possible that the N-terminal fALS-associated mutations disrupt a putative second NLS. However, our mutational analysis (Figure 2D and E; Supplementary Figure S2A and B) suggests that N-terminal fALS mutations act through a different cellular mechanism. As the SYGQ- and G-rich domains are involved in transcriptional activation and have been shown to interact with RNA polymerase II and various transcription factors (Zinszner et al, 1994; Yang et al, 2000; Law et al, 2006), the mutations instead might modulate FUS function as a transcriptional activator, either impairing or aberrantly activating transcription of neuronal target genes. The finding that different clusters of mutations within one gene may affect different cellular mechanisms is not too surprising, given the fact that familial $\mathrm{AD}$-causing mutations in three different regions within and around the amyloid $\beta(A \beta)$ peptide domain have fundamentally different consequences on $A \beta$ metabolism and aggregation (Haass, 2004).

Strikingly, we found a co-deposition of cytosolic FUS with various stress granule marker proteins in cells subjected to cellular stress as well as in brains of fALS-FUS and FTLD-FUS patients. However, some differences exist between the FUSpositive stress granules observed in our cellular models (primary neurons and HeLa cells) and the FUS inclusions present in fALS and FTLD-FUS patients: GFP-M9M-expressing cells or cells exposed to heat shock contain multiple small FUS granules in the perinuclear region or in neuritic processes (Figures 6 and 8). In contrast, NCIs in brains of FTLD-FUS patients are usually much larger (see Figure 7; Neumann et al, 2009a). However, the granules in our in vitro cultures and the inclusions in brains of patients also share important properties: first, they resemble each other ultrastructurally, as both stress granules and inclusions in BIBID and NIFID patients appear as fibrillogranular aggregates in electron microscopy (Munoz-Garcia and Ludwin, 1984; Mosaheb et al, 2005; Souquere et al, 2009). A second common property is their protein composition: they both contain FUS and the stress granule markers PABP-1 and eIF4G, but not TDP-43. Interestingly, before the discovery of FUS in ALS and FTLD, Fujita et al (2008) described that the basophilic inclusions in BIBD patients contain markers of stress granules. Stress granules are known to be dynamic entities that can enlarge and coalesce on prolonged stress exposure (Kedersha et al, 2000). Hence, it seems possible that in the presence of chronic stress, such as oxidative stress, viral infections or proteasome inhibition (Anderson and Kedersha, 2008), small stress granules give rise to larger granules and eventually to large, insoluble inclusions. It will, therefore, be interesting to see if indeed RNA and further RNA-binding proteins commonly found in stress granules in in vitro cultures are present in FUS inclusions in fALS and FTLD-FUS patients.

Several reports recently described that TDP-43 can be found in stress granules under various experimental condi- tions (Colombrita et al, 2009; Moisse et al, 2009; Volkening et al, 2009; Freibaum et al, 2010). First, sciatic axotomy in adult mice was reported to cause a marked increase of cytoplasmic TDP-43 and its co-localization with the RNAbinding proteins Staufen and TIA-1 (Moisse et al, 2009). In cellular models, oxidative stress and proteasome inhibition were reported to lead to a partial recruitment of TDP-43 into stress granules (Colombrita et al, 2009; Freibaum et al, 2010), and Freibaum et al identified numerous components of stress granules as TDP-43-interacting proteins (Freibaum et al, 2010). Although our data clearly show that the FUS-containing stress granules observed after GFP-M9M expression or heat shock do not contain TDP-43 (Figures 5D and 8C), this might be explained by the fact that TDP-43 was predominantly nuclear under these experimental conditions. According to our two hit model, it seems possible that similar to FUS, cytosolic relocalization of TDP-43 is a prerequisite for efficient stress granule recruitment. Thus, it can be speculated that in the studies mentioned above, TDP-43 was at least partially mislocalized to the cytosol before cellular stress.

What remains controversial is the data on stress granule markers in patients with TDP-43 pathology. One study reported a stronger TIA-1 (stress granule) and XRN-1 (P body) staining in sALS patients compared with healthy controls (Volkening et al, 2009), whereas another study found a lack of stress granule markers in TDP-43 inclusions of sALS patients (Colombrita et al, 2009). Consistent with the latter study, inclusions of FTLD-TDP patients were consistently negative for the stress granule markers PABP-1 and eIF4G in our study (Figure 7). Further studies with larger number of patients are needed to clarify this issue.

Although we did not observe HA-tagged WT-FUS (Figure $8 \mathrm{~A}$ and $\mathrm{B}$ ) or endogenous FUS (data not shown) in stress granules on heat shock, it is possible that very small amounts of FUS, undetectable by our antibodies, are present in stress granules even in untransfected or FUS-WT-transfected cells. That this might be the case is suggested by the report of Andersson et al (2008), showing a recruitment of endogenous FUS to stress granules on exposure of cell lines to oxidative stress. Nevertheless, our analysis of fALS-associated FUS mutations strongly suggests that cytosolic mislocalization of FUS favours recruitment of the protein into stress granules, as the degree of stress granule recruitment correlated with the cytoplasmic mislocalization of the individual point mutations (Figure 8A). Thus, our data suggest that cytosolic FUS is recruited to stress granules, and furthermore, imply that an increased presence of FUS in the cytosol favours the formation of FUS-positive stress granules. In the light of these data, one may postulate that at least small amounts of FUS have to accumulate in the cytosol to trigger the onset of sporadic FTLD-FUS. As not only fALS patients with FUS mutations but also sporadic FTLD-FUS patients show a neuronal cytoplasmic redistribution of FUS (Munoz et al, 2009; Neumann et al, 2009a,b) and a co-deposition of FUS and stress granule marker proteins in NCIs (this study), the question arises what causes the abnormal cytoplasmic accumulation of FUS in cases without FUS mutations. Subtle alterations in the Trp pathway, for example caused by reduced Trp expression or post-translational modifications of FUS, might lead to an increase of FUS in the cytosol even in the absence of FUS mutations. Indeed, weak cytosolic FUS staining is 
consistently observed in post-mortem brain tissue from healthy controls (Neumann et al, 2009a), indicating that cytosolic FUS may accumulate during ageing. In combination with environmental stress, a small increase in cytosolic FUS may then be sufficient to initiate clustering of FUS in stress granules and eventually larger inclusions (Figure 9).

\section{Materials and methods}

\section{Cell culture and transfection}

Human cervical carcinoma cells (HeLa) were cultured in Dulbecco's modified Eagle's medium with Glutamax (Invitrogen) supplemented with $10 \%$ (vol/vol) foetal calf serum (FCS, Invitrogen) and penicillin/streptomycin (PAA). Transfection of HeLa cells was carried out with Fugene 6 (Roche) or Lipofectamine 2000 (Invitrogen) according to the manufacturer's instructions. Hippocampal and cortical neurons were cultured from embryonic day 19 rat embryos as described earlier (Tada et al, 2007). Neurons were transfected on day in vitro (DIV) 5 using Lipofectamine 2000 (Invitrogen) and were analysed on DIV 7. When mCherry was used as a filler to visualize neuron morphology, the mCherry:HA-FUS DNA ratio was $1: 10$

\section{Antibodies and inhibitors}

A complete list of all antibodies used can be found in the Supplementary data. Cycloheximide (Sigma) was used at a concentration of $20 \mu \mathrm{g} / \mathrm{ml}$.

\section{cDNA constructs and primers}

The cDNA sequence of human FUS (NM_004960) was amplified from a human brain cDNA library and was cloned by XhoI/BamHI restriction digest into the pcDNA3.1/Hygro(-) vector (Invitrogen) with an N-terminal HA-tag. HA-FUS-P525L was generated from the FUS-WT construct by QuikChange mutagenesis (Stratagene), all other FUS mutations were introduced through conventional PCR primers and PCR products were cloned into pcDNA3.1/Hygro(-) through restriction digest. The pGST-EGFP-C1 vector was generated by inserting the GST sequence with a Kozak sequence into the NheI/AgeI restriction sites of the pEGFP-C1 vector (Clontech). NLS reporter constructs were generated by ligating annealed oligos into the Xhol/BamHI restriction sites of pGST-EGFP-C1. The nuclear import inhibitor constructs (GFP-M9M, GFP-Bimax2) were generated by ligating annealed oligos into the Xhol/BamHI restriction sites of pEGFP-C1. For all constructs, sequence integrity was verified by sequencing. Oligonucleotides sequences are available on request.

\section{Zebrafish husbandry and embryo injection}

All of the experiments were performed in compliance with the guidelines of the German Council on Animal Care. WT AB zebrafish were kept at $28^{\circ} \mathrm{C}$ and raised and mated as described (Mullins et al, 1994). GST-EGFP constructs $(25 \mathrm{ng} / \mu \mathrm{l})$ were injected into fertilized eggs at the one-cell stage; $48 \mathrm{~h}$ old embryos were stained as described in the Supplementary data.

\section{Human post-mortem tissue}

Cases with confirmed FUS pathology used in this study have been earlier described and included a case of fALS with an FUS-R521C mutation (Rademakers et al, 2010) and cases of aFTLD-U $(n=3)$ (Neumann et al, 2009a), NIFID ( $n=3$ ) (Neumann et al, 2009b) and $\operatorname{BIBD}(n=1)$ (Munoz et al, 2009). In addition, FTLD-TDP $(n=2)$ as neurologic controls and cases with no history of neurologic diseases $(n=2)$ were included.

\section{Immunocytochemistry and immunohistochemistry}

For immunocytochemistry on HeLa cells and neurons, cells were fixed for $15 \mathrm{~min}$ in $4 \%$ paraformaldehyde in PBS, permeabilized for 5 min in $0.2 \%$ Triton X-100 with $50 \mathrm{mM} \mathrm{NH}_{4} \mathrm{Cl}$ and subsequently blocked for $20-30 \mathrm{~min}$ in blocking buffer ( $5 \%$ goat serum or $2 \%$ BSA in PBSS $=$ PBS with $0.1 \%$ saponin). Cells were stained with the indicated primary and secondary antibodies diluted in blocking buffer for $30 \mathrm{~min}$ and were washed $5 \times$ in PBSS. To visualize nuclei, cells were stained with TO-PRO-3 iodide (Invitrogen) for $15 \mathrm{~min}$ and were washed $3 \times$ in PBS. Coverslips were mounted onto glass slides using ProLong Gold Antifade Reagent (Invitrogen). All steps were carried out at RT.

Immunohistochemistry on human post-mortem material was performed on $5 \mu \mathrm{m}$ thick sections of formalin fixed, paraffinembedded tissue from spinal cord, medulla or hippocampus with the indicated antibodies (after microwave antigen retrieval) and the avidin-biotin complex detection system (Vector Laboratories) with $3,3^{\prime}$-diaminobenzidine as chromogen. Double-label immunofluorescence for PABP-1 and p62 (after microwave antigen retrieval) was performed using Alexa-488 and -594-conjugated secondary antibodies; 4'-6-diamidino-2-phenylindol (Vector Laboratories) was used for nuclear counter-staining.

\section{Image acquisition and quantification}

Confocal images of HeLa cells, primary neurons and zebrafish embryos were obtained with an inverted laser scanning confocal microscope (Zeiss Axiovert $200 \mathrm{M}$ ) with a $63 \times / 1.4$ NA oil immersion lens, using a pinhole diameter of 1 Airy unit. Pictures were taken and analysed with the LSM 510 confocal software (Zeiss), and, if necessary, for printing, brightness and contrast were linearly enhanced using the LSM image browser (Zeiss). For HeLa cells, single confocal images were taken in the plane of the largest cytosolic area. For neuronal cultures and zebrafish embryos, a series of images along the $z$ axis was taken and projected into a single image using the maximal projection tool of the LSM 510 software. Immunofluorescence images of brain sections were obtained by wide-field fluorescence microscopy (BX61 Olympus with digital camera F-view, Olympus).

Nuclear and cytosolic localization was quantified with the LSM 510's co-localization tool as follows: total fluorescence intensities of the green channel were calculated from the mean fluorescence intensity and the number of pixels. Pixels that were co-localized with TO-PRO-3 were considered 'nuclear' and pixels that did not overlap with TO-PRO-3 were considered 'cytosolic'. For each sample, 7-12 randomly selected fields were analysed, containing a total of 50-100 transfected cells. Means across all fields were calculated and s.d. are indicated by error bars. Pictures and quantification shown are from one experiment, but are representative of several experiments.

\section{siRNA-mediated knockdown of Trp1 and Trp2}

Trp1/2 knockdown was achieved using two different siRNA pools: Trp1/2 pool \#1 consisted of the Trp1-specific siGENOME siRNA D011308-01 (target sequence: $5^{\prime}$-guauagagaugcagccuua- $3^{\prime}$ ) and the Trp2-specific siGENOME SMART pool M-020491-01 (target sequences: $5^{\prime}$-gggcagagaugcagccuua- $3^{\prime} ; 5^{\prime}$-gcaguucucugagcaauuc-3'; $5^{\prime}$-aaacaggagugucucaaca- $3^{\prime} ; \quad 5^{\prime}$-gcgcugauggacaauauug- $\left.3^{\prime}\right), \quad$ both from Dharmacon. Trp1/2 pool $\# 2$ consisted of the Trp1-specific siGENOME siRNA D-011308-04 from Dharmacon (target sequence: $5^{\prime}$-caauuggucgucuugguua- $3^{\prime}$ ) and the Trp2-specific siGENOME SMART pool M-020491-01 (see above). A non-targeting (NT) siRNA (ON-TARGET plus NT siRNA \#3, D-001810-03 from Dharmacon) was used as a negative control. Cells were reverse transfected using a total of $50 \mathrm{pmol}$ siRNA and $5 \mu \mathrm{l}$ Lipofectamine 2000 (Invitrogen) per six well. Medium was changed $4-6 \mathrm{~h}$ post-transfection and effect of knockdown was analysed $48-72 \mathrm{~h}$ post-transfection.

\section{Cell lysates and immunoblotting}

Cells were washed twice in PBS, scraped off and pelleted at $1000 \mathrm{~g}$, $5 \mathrm{~min}$. Total cell lysates were prepared in ice cold RIPA buffer freshly supplemented with complete EDTA-free protease inhibitor cocktail (Roche). After $15 \mathrm{~min}$ lysis on ice, lysates were sonicated in a bioruptor (Diagenode, $45 \mathrm{~s}$ on high) and protein concentration was determined by BCA protein assay (Pierce); $4 \times$ SDS-PAGE sample buffer was added and samples were boiled for $5 \mathrm{~min}$. Proteins were separated by SDS-PAGE, transferred onto a PVDF membrane (Immobilon-P, Millipore) and analysed by immunoblotting using the indicated antibodies. Bound antibodies were detected with the chemiluminescence detection reagents ECL (Amersham) or Immobilon (Millipore)

\section{Structural modelling}

The C-terminal amino-acid residues D520-Y526 of FUS were modelled manually into the Trp-binding pocket. Residues of the non-classical NLSs of subtype RXXPY (pdb-entries 2OT8 (Cansizoglu et al, 2007) and 2Z5K (Imasaki et al, 2007) differing between the experimental structures and the FUS C-terminal sequence were 
exchanged obeying standard amino-acid conformations (Engh and Huber, 1991) and the resulting structure was locally energy minimized using MAIN (Turk, 1992), keeping the Trp molecule rigid.

\section{Supplementary data}

Supplementary data are available at The EMBO Journal Online (http://www.embojournal.org).

\section{Acknowledgements}

We thank Drs Harald Steiner, Richard Page and Stefan Lichtenthaler for critical discussion, Dr Sven Lammich for providing human brain cDNA and the Hans and Ilse Breuer Foundation for the confocal microscope. This work was supported by the Center for Integrated

\section{References}

Anderson P, Kedersha N (2006) RNA granules. J Cell Biol 172: 803-808

Anderson P, Kedersha N (2008) Stress granules: the Tao of RNA triage. Trends Biochem Sci 33: 141-150

Andersson MK, Stahlberg A, Arvidsson Y, Olofsson A, Semb $\mathrm{H}$, Stenman G, Nilsson O, Aman P (2008) The multifunctional FUS, EWS and TAF15 proto-oncoproteins show cell type-specific expression patterns and involvement in cell spreading and stress response. BMC Cell Biol 9: 37-53

Arai T, Hasegawa M, Akiyama H, Ikeda K, Nonaka T, Mori H, Mann D, Tsuchiya K, Yoshida M, Hashizume Y, Oda T (2006) TDP-43 is a component of ubiquitin-positive tau-negative inclusions in frontotemporal lobar degeneration and amyotrophic lateral sclerosis. Biochem Biophys Res Commun 351: 602-611

Ayala YM, Zago P, D’Ambrogio A, Xu YF, Petrucelli L, Buratti E, Baralle FE (2008) Structural determinants of the cellular localization and shuttling of TDP-43. J Cell Sci 121: 3778-3785

Belzil VV, Valdmanis PN, Dion PA, Daoud H, Kabashi E, Noreau A, Gauthier J, Hince P, Desjarlais A, Bouchard JP, Lacomblez L, Salachas F, Pradat PF, Camu W, Meininger V, Dupre N, Rouleau GA (2009) Mutations in FUS cause FALS and SALS in French and French Canadian populations. Neurology 73: 1176-1179

Boillee S, Vande Velde C, Cleveland DW (2006) ALS: a disease of motor neurons and their nonneuronal neighbors. Neuron 52: 39-59

Buratti E, Baralle FE (2008) Multiple roles of TDP-43 in gene expression, splicing regulation, and human disease. Front Biosci 13: $867-878$

Cansizoglu AE, Lee BJ, Zhang ZC, Fontoura BM, Chook YM (2007) Structure-based design of a pathway-specific nuclear import inhibitor. Nat Struct Mol Biol 14: 452-454

Chio A, Restagno G, Brunetti M, Ossola I, Calvo A, Mora G, Sabatelli M, Monsurro MR, Battistini S, Mandrioli J, Salvi F, Spataro R, Schymick J, Traynor BJ, La Bella V (2009) Two Italian kindreds with familial amyotrophic lateral sclerosis due to FUS mutation. Neurobiol Aging 30: 1272-1275

Colombrita C, Zennaro E, Fallini C, Weber M, Sommacal A, Buratti E, Silani V, Ratti A (2009) TDP-43 is recruited to stress granules in conditions of oxidative insult. J Neurochem 111: 1051-1061

Corrado L, Del Bo R, Castellotti B, Ratti A, Cereda C, Penco S, Soraru G, Carlomagno Y, Ghezzi S, Pensato V, Colombrita C, Gagliardi S, Cozzi L, Orsetti V, Mancuso M, Siciliano G, Mazzini L, Comi GP, Gellera C, Ceroni M et al (2010) Mutations of FUS gene in sporadic amyotrophic lateral sclerosis. J Med Genet 47: 190-194

DeJesus-Hernandez M, Kocerha J, Finch N, Crook R, Baker M, Desaro P, Johnston A, Rutherford N, Wojtas A, Kennelly K, Wszolek ZK, Graff-Radford N, Boylan K, Rademakers R (2010) De novo truncating FUS gene mutation as a cause of sporadic amyotrophic lateral sclerosis. Human Mutat 31: E1377-E1389

Drepper C, Herrmann T, Wessig C, Beck M, Sendtner M (2009) Cterminal FUS/TLS mutations in familial and sporadic ALS in Germany. Neurobiol Aging (advance online publication)

Duering M, Grimm MO, Grimm HS, Schroder J, Hartmann T (2005) Mean age of onset in familial Alzheimer's disease is determined by amyloid beta 42 . Neurobiol Aging 26: 785-788
Protein Science Munich (CIPSM), the Competence Network of Neurodegenerative Diseases (KNDD) of the Bundesministerium für Bildung und Forschung (BMBF), the Sonderforschungsbereich Molecular Mechanisms of Neurodegeneration (SFB 596) and an EMBO postdoctoral fellowship (to DD). DE is supported by the Helmholtz Young Investigator Program. MN is supported by the Stavros-Niarchos Foundation and the Synapsis Foundation. IRAM is supported by the Canadian Institutes of Health Research and the Pacific Alzheimer's Research Foundation. $\mathrm{CH}$ is supported by a 'Forschungsprofessur' of the Ludwig-Maximilians University.

\section{Conflict of interest}

The authors declare that they have no conflict of interest.

Engh RA, Huber R (1991) Accurate bond and angle parameters for X-ray protein-structure refinement. Acta Crystallogr A 47: $329-400$

Freibaum BD, Chitta RK, High AA, Taylor JP (2010) Global analysis of TDP-43 interacting proteins reveals strong association with RNA splicing and translation machinery. J Proteome Res 9: $1104-1120$

Fujii R, Okabe S, Urushido T, Inoue K, Yoshimura A, Tachibana T, Nishikawa T, Hicks GG, Takumi T (2005) The RNA binding protein TLS is translocated to dendritic spines by mGluR5 activation and regulates spine morphology. Curr Biol 15: 587-593

Fujii R, Takumi T (2005) TLS facilitates transport of mRNA encoding an actin-stabilizing protein to dendritic spines. J Cell Sci 118: $5755-5765$

Fujita K, Ito H, Nakano S, Kinoshita Y, Wate R, Kusaka H (2008) Immunohistochemical identification of messenger RNA-related proteins in basophilic inclusions of adult-onset atypical motor neuron disease. Acta Neuropathol 116: 439-445

Gitcho MA, Baloh RH, Chakraverty S, Mayo K, Norton JB, Levitch D, Hatanpaa KJ, White III CL, Bigio EH, Caselli R, Baker M, AlLozi MT, Morris JC, Pestronk A, Rademakers R, Goate AM, Cairns NJ (2008) TDP-43 A315T mutation in familial motor neuron disease. Ann Neurol 63: 535-538

Guttinger S, Muhlhausser P, Koller-Eichhorn R, Brennecke J, Kutay $\mathrm{U}$ (2004) Transportin2 functions as importin and mediates nuclear import of HuR. Proc Natl Acad Sci USA 101: 2918-2923

Haass C (2004) Take five-BACE and the gamma-secretase quartet conduct Alzheimer's amyloid beta-peptide generation. EMBO J 23: $483-488$

Harley VR, Layfield S, Mitchell CL, Forwood JK, John AP, Briggs LJ, McDowall SG, Jans DA (2003) Defective importin beta recognition and nuclear import of the sex-determining factor SRY are associated with XY sex-reversing mutations. Proc Natl Acad Sci USA 100: 7045-7050

Iijima M, Suzuki M, Tanabe A, Nishimura A, Yamada M (2006) Two motifs essential for nuclear import of the hnRNP A1 nucleocytoplasmic shuttling sequence M9 core. FEBS Lett 580: 1365-1370

Imasaki T, Shimizu T, Hashimoto H, Hidaka Y, Kose S, Imamoto N, Yamada M, Sato M (2007) Structural basis for substrate recognition and dissociation by human transportin 1. Mol Cell 28: 57-67

Johnson BS, Snead D, Lee JJ, McCaffery JM, Shorter J, Gitler AD (2009) TDP-43 is intrinsically aggregation-prone, and amyotrophic lateral sclerosis-linked mutations accelerate aggregation and increase toxicity. J Biol Chem 284: 20329-20339

Kabashi E, Valdmanis PN, Dion P, Spiegelman D, McConkey BJ, Vande Velde C, Bouchard JP, Lacomblez L, Pochigaeva K, Salachas F, Pradat PF, Camu W, Meininger V, Dupre N, Rouleau GA (2008) TARDBP mutations in individuals with sporadic and familial amyotrophic lateral sclerosis. Nat Genet 40: 572-574

Kalderon D, Roberts BL, Richardson WD, Smith AE (1984) A short amino acid sequence able to specify nuclear location. Cell 39: 499-509

Kedersha N, Anderson P (2007) Mammalian stress granules and processing bodies. Methods Enzymol 431: 61-81

Kedersha N, Cho MR, Li W, Yacono PW, Chen S, Gilks N, Golan DE, Anderson P (2000) Dynamic shuttling of TIA-1 accompanies the 
recruitment of mRNA to mammalian stress granules. J Cell Biol 151: $1257-1268$

Kiebler MA, Bassell GJ (2006) Neuronal RNA granules: movers and makers. Neuron 51: 685-690

Kosugi S, Hasebe M, Entani T, Takayama S, Tomita M, Yanagawa H (2008) Design of peptide inhibitors for the importin alpha/beta nuclear import pathway by activity-based profiling. Chem Biol 15: 940-949

Kwiatkowski Jr TJ, Bosco DA, Leclerc AL, Tamrazian E, Vanderburg CR, Russ C, Davis A, Gilchrist J, Kasarskis EJ, Munsat T, Valdmanis P, Rouleau GA, Hosler BA, Cortelli P, de Jong PJ, Yoshinaga Y, Haines JL, Pericak-Vance MA, Yan J, Ticozzi N et al (2009) Mutations in the FUS/TLS gene on chromosome 16 cause familial amyotrophic lateral sclerosis. Science 323: 1205-1208

Lagier-Tourenne C, Cleveland DW (2009) Rethinking ALS: the FUS about TDP-43. Cell 136: 1001-1004

Lai SL, Abramzon Y, Schymick JC, Stephan DA, Dunckley T, Dillman A, Cookson M, Calvo A, Battistini S, Giannini F, Caponnetto C, Mancardi GL, Spataro R, Monsurro MR, Tedeschi G, Marinou K, Sabatelli M, Conte A, Mandrioli J, Sola P et al (2010) FUS mutations in sporadic amyotrophic lateral sclerosis Neurobiol Aging (advance online publication)

Law WJ, Cann KL, Hicks GG (2006) TLS, EWS and TAF15: a model for transcriptional integration of gene expression. Brief Funct Genomic Proteomic 5: 8-14

Lee BJ, Cansizoglu AE, Suel KE, Louis TH, Zhang Z, Chook YM (2006) Rules for nuclear localization sequence recognition by karyopherin beta 2. Cell 126: 543-558

Li B, Zhang W, Chan G, Jancso-Radek A, Liu S, Weiss MA (2001) Human sex reversal due to impaired nuclear localization of SRY. A clinical correlation. J Biol Chem 276: 46480-46484

Mackenzie IR, Bigio EH, Ince PG, Geser F, Neumann M, Cairns NJ, Kwong LK, Forman MS, Ravits J, Stewart H, Eisen A, McClusky L, Kretzschmar HA, Monoranu CM, Highley JR, Kirby J, Siddique T, Shaw PJ, Lee VM, Trojanowski JQ (2007) Pathological TDP-43 distinguishes sporadic amyotrophic lateral sclerosis from amyotrophic lateral sclerosis with SOD1 mutations. Ann Neurol 61: 427-434

Mackenzie IR, Neumann M, Bigio EH, Cairns NJ, Alafuzoff I, Kril J, Kovacs GG, Ghetti B, Halliday G, Holm IE, Ince PG, Kamphorst W, Revesz T, Rozemuller AJ, Kumar-Singh S, Akiyama H, Baborie A, Spina S, Dickson DW, Trojanowski JQ et al (2010) Nomenclature and nosology for neuropathologic subtypes of frontotemporal lobar degeneration: an update. Acta Neuropathol 119: 1-4

Mackenzie IR, Rademakers R (2008) The role of transactive response DNA-binding protein-43 in amyotrophic lateral sclerosis and frontotemporal dementia. Curr Opin Neurol 21: 693-700

Moisse K, Volkening K, Leystra-Lantz C, Welch I, Hill T, Strong MJ (2009) Divergent patterns of cytosolic TDP-43 and neuronal progranulin expression following axotomy: implications for TDP-43 in the physiological response to neuronal injury. Brain Res 1249: 202-211

Mosaheb S, Thorpe JR, Hashemzadeh-Bonehi L, Bigio EH, Gearing M, Cairns NJ (2005) Neuronal intranuclear inclusions are ultrastructurally and immunologically distinct from cytoplasmic inclusions of neuronal intermediate filament inclusion disease. Acta Neuropathol 110: 360-368

Mullins MC, Hammerschmidt M, Haffter P, Nusslein-Volhard C (1994) Large-scale mutagenesis in the zebrafish: in search of genes controlling development in a vertebrate. Curr Biol 4: 189-202

Munoz-Garcia D, Ludwin SK (1984) Classic and generalized variants of Pick's disease: a clinicopathological, ultrastructural, and immunocytochemical comparative study. Ann Neurol 16: 467-480

Munoz DG, Neumann M, Kusaka H, Yokota O, Ishihara K, Terada S, Kuroda S, Mackenzie IR (2009) FUS pathology in basophilic inclusion body disease. Acta Neuropathol 118: 617-627

Neumann M, Igaz LM, Kwong LK, Nakashima-Yasuda $H$, Kolb SJ, Dreyfuss G, Kretzschmar HA, Trojanowski JQ, Lee VM (2007) Absence of heterogeneous nuclear ribonucleoproteins and survival motor neuron protein in TDP-43 positive inclusions in frontotemporal lobar degeneration. Acta Neuropathol 113: 543-548

Neumann M, Rademakers R, Roeber S, Baker M, Kretzschmar HA, Mackenzie IR (2009a) A new subtype of frontotemporal lobar degeneration with FUS pathology. Brain 132: 2922-2931
Neumann M, Roeber S, Kretzschmar HA, Rademakers R, Baker M, Mackenzie IR (2009b) Abundant FUS-immunoreactive pathology in neuronal intermediate filament inclusion disease. Acta Neuropathol 118: 605-616

Neumann M, Sampathu DM, Kwong LK, Truax AC, Micsenyi MC, Chou TT, Bruce J, Schuck T, Grossman M, Clark CM, McCluskey LF, Miller BL, Masliah E, Mackenzie IR, Feldman H, Feiden W, Kretzschmar HA, Trojanowski JQ, Lee VM (2006) Ubiquitinated TDP-43 in frontotemporal lobar degeneration and amyotrophic lateral sclerosis. Science 314: 130-133

Nonaka T, Kametani F, Arai T, Akiyama H, Hasegawa M (2009) Truncation and pathogenic mutations facilitate the formation of intracellular aggregates of TDP-43. Hum Mol Genet 18: 3353-3364

Page RM, Baumann K, Tomioka M, Perez-Revuelta BI, Fukumori A, Jacobsen H, Flohr A, Luebbers T, Ozmen L, Steiner H, Haass C (2008) Generation of Abeta38 and Abeta42 is independently and differentially affected by familial Alzheimer disease-associated presenilin mutations and gamma-secretase modulation. J Biol Chem 283: 677-683

Rademakers R, Stewart H, DeJesus-Hernandez M, Krieger C, GraffRadford N, Fabros M, Briemberg H, Cashman N, Eisen A, Mackenzie IRA (2010) FUS gene mutations in familial and sporadic amyotrophic lateral sclerosis. Muscle Nerve (advance online publication)

Rebane A, Aab A, Steitz JA (2004) Transportins 1 and 2 are redundant nuclear import factors for hnRNP A1 and HuR. RNA 10: $590-599$

Souquere S, Mollet S, Kress M, Dautry F, Pierron G, Weil D (2009) Unravelling the ultrastructure of stress granules and associated Pbodies in human cells. J Cell Sci 122: 3619-3626

Sreedharan J, Blair IP, Tripathi VB, Hu X, Vance C, Rogelj B, Ackerley S, Durnall JC, Williams KL, Buratti E, Baralle F, de Belleroche J, Mitchell JD, Leigh PN, Al-Chalabi A, Miller CC, Nicholson G, Shaw CE (2008) TDP-43 mutations in familial and sporadic amyotrophic lateral sclerosis. Science 319: $1668-1672$

Suzuki N, Aoki M, Warita H, Kato M, Mizuno H, Shimakura N, Akiyama T, Furuya H, Hokonohara T, Iwaki A, Togashi S, Konno H, Itoyama Y (2010) FALS with FUS mutation in Japan, with early onset, rapid progress and basophilic inclusion. J Hum Genet 55: 252-254

Tada T, Simonetta A, Batterton M, Kinoshita M, Edbauer D, Sheng M (2007) Role of Septin cytoskeleton in spine morphogenesis and dendrite development in neurons. Curr Biol 17: 1752-1758

Talbot K, Ansorge O (2006) Recent advances in the genetics of amyotrophic lateral sclerosis and frontotemporal dementia: common pathways in neurodegenerative disease. Hum Mol Genet 15 (Spec No 2): R182-R187

Terry LJ, Shows EB, Wente SR (2007) Crossing the nuclear envelope: hierarchical regulation of nucleocytoplasmic transport. Science 318: 1412-1416

Ticozzi N, Silani V, LeClerc AL, Keagle P, Gellera C, Ratti A, Taroni F, Kwiatkowski Jr TJ, McKenna-Yasek DM, Sapp PC, Brown Jr RH, Landers JE (2009) Analysis of FUS gene mutation in familial amyotrophic lateral sclerosis within an Italian cohort. Neurology 73: $1180-1185$

Turk D (1992) Weiterentwicklung eines Programms für Molekülgraphik und Elektronendichte-Manipulation und seine Anwendung auf verschiedene Proteinstrukturaufklärungen. Munich, Germany: Technische Universität München, PhD thesis

Valdmanis PN, Rouleau GA (2008) Genetics of familial amyotrophic lateral sclerosis. Neurology 70: 144-152

Vance C, Rogelj B, Hortobagyi T, De Vos KJ, Nishimura AL, Sreedharan J, Hu X, Smith B, Ruddy D, Wright P, Ganesalingam J, Williams KL, Tripathi V, Al-Saraj S, Al-Chalabi A, Leigh PN, Blair IP, Nicholson G, de Belleroche J, Gallo JM et al (2009) Mutations in FUS, an RNA processing protein, cause familial amyotrophic lateral sclerosis type 6. Science 323: 1208-1211

Volkening $\mathrm{K}$, Leystra-Lantz C, Yang W, Jaffee H, Strong MJ (2009) Tar DNA binding protein of $43 \mathrm{kDa}$ (TDP-43), 14-3-3 proteins and copper/zinc superoxide dismutase (SOD1) interact to modulate NFL mRNA stability. Implications for altered RNA processing in amyotrophic lateral sclerosis (ALS). Brain Res 1305: $168-182$

Winton MJ, Igaz LM, Wong MM, Kwong LK, Trojanowski JQ, Lee VM (2008) Disturbance of nuclear and cytoplasmic TAR DNA- 
binding protein (TDP-43) induces disease-like redistribution, sequestration, and aggregate formation. $J$ Biol Chem 283: 13302-13309

Yang L, Embree LJ, Hickstein DD (2000) TLS-ERG leukemia fusion protein inhibits RNA splicing mediated by serine-arginine proteins. Mol Cell Biol 20: 3345-3354

Zakaryan RP, Gehring H (2006) Identification and characterization of the nuclear localization/retention signal in the EWS protooncoprotein. J Mol Biol 363: 27-38
Zinszner H, Albalat R, Ron D (1994) A novel effector domain from the RNA-binding protein TLS or EWS is required for oncogenic transformation by CHOP. Genes Dev 8: 2513-2526

Zinszner H, Immanuel D, Yin Y, Liang FX, Ron D (1997a) A topogenic role for the oncogenic N-terminus of TLS: nucleolar localization when transcription is inhibited. Oncogene 14: 451-461

Zinszner H, Sok J, Immanuel D, Yin Y, Ron D (1997b) TLS (FUS) binds RNA in vivo and engages in nucleo-cytoplasmic shuttling. J Cell Sci 110 (Pt 15): 1741-1750 